\title{
Optimal and robust control of streaks in pipe flow
}

\author{
By M. I. GAVARINI, ${ }^{1}$ A. BOTTARO ${ }^{2}$ \\ AND F. T. M. NIEUWSTADT ${ }^{1} \dagger$ \\ ${ }^{1}$ J. M. Burgers Centre, Delft University of Technology, 2628 CA Delft, The Netherlands \\ ${ }^{2}$ DIAM, Università di Genova, Via Montallegro 1, 16145 Genova, Italy
}

(Received 1 April 2004 and in revised form 21 February 2005)

Control theory is used to determine optimal disturbances in pipe flow and the forcing, in the form of blowing and suction at the wall, capable of attenuating them. An approach is adopted, based on a parabolic approximation of the linear Navier-Stokes equations, which is appropriate when dealing with asymptotically elongated (in the streamwise direction) flow structures. A cost functional is introduced and maximized in the optimal-perturbation problem or minimized, for a given inflow perturbation, in the optimal-control problem. The extrema of the cost functional are reached by means of an iterative technique, based on the numerical solution of the equations for the state and the adjoint state, coupled via transfer and optimality conditions. Central to the control is the determination of the Green's function expressing the receptivity of the flow to wall forcing. A considerable reduction in output disturbance energy, as compared to the uncontrolled case, is obtained for control laws operating both over a long section of the pipe or over shorter strips. Finally, a robust control is sought, by simultaneously computing the worst inflow condition and the corresponding best control at the wall.

\section{Introduction}

In wall-bounded shear flows, elongated streaks of low and high streamwise velocity amplified via a process called transient growth - are known to play an important role in the initial stages of transition (see, for instance, Schmid \& Henningson 2001). From a mathematical point of view, transient growth is the consequence of the non-normality of the linearized operator governing the evolution of infinitesimal disturbances (Trefethen et al. 1993). From a physical point of view, it is connected to the displacement of fluid elements in the direction of the mean gradient by a wall-normal velocity perturbation, such that their horizontal momentum is conserved. Hence it is also known as the lift-up effect (Landahl 1980).

One of the early studies on the role of transient growth in the transition to turbulence in cylindrical pipe flow, was performed by Boberg \& Brosa (1988). They were also the first to determine those initial disturbances, subsequently defined 'optimal', that undergo the largest possible amplification over a given time interval. These authors recognized that a mechanism permitting chaotic motion in a linearly stable system, must rely on the non-normality of the linearized operator, i.e. on the near collinearity of its eigenfunctions, although they also stressed the importance of nonlinearities to sustain the process. In their work, a picture of the mechanism underlying

$\dagger$ Professor Nieuwstadt died on 18 May 2005, during the publication process of this paper. 
transition is drawn in terms of two systems called 'mothers' and 'daughters': small disturbances (the mothers) induce stronger disturbances (the daughters) that possess and dissipate almost all the (disturbance) energy. The mothers feed the daughters by controlling the transfer of energy from the basic flow, but they cannot incorporate this energy for their own benefit. As a result, the mothers decay and as a consequence the daughters fade away also. This is where the effect of nonlinearity must come into play, because its role is to transform part of the daughters back into mothers so that the whole sequence can start over again. By looking at the maximum exploitation of the linear transfer of energy, the authors were able to isolate an 'ideal' (or 'optimal') mother, in the form of a streamwise elongated vortex. The daughters are then found to be streamwise oriented low- and high-speed streaks.

Subsequently, transient growth in Hagen-Poiseuille flow has been analysed, within the framework of temporal stability theory, by Bergström (1993), Schmid \& Henningson (1994) and Trefethen et al. (1993). They all used the method described by Farrell (1988) who determined optimal initial conditions in a temporal setting for viscous shear flows. To obtain this optimal initial disturbance, we first consider a generic perturbation as a weighted sum of eigenvectors of the linear problem. The weights are then determined to provide the condition of maximum gain over a given time span. This can be accomplished either by application of a variational technique, that leads to the solution of a generalized eigenvalue problem (see Butler \& Farrell 1992) or directly by means of singular-value decomposition (see Schmid \& Henningson 1994, 2001). As indicated above, this (temporal) optimal disturbance resembles a streamwise vortex, which diverts the mean flow energy into streaks of low and high streamwise velocity.

Reshotko \& Tumin (2001) have recently analysed optimal disturbances in the framework of spatial stability theory, which is physically more relevant because it allows for a direct comparison with experimental results. However, the method proposed by Farrell (1988) cannot be applied here. The elliptic system describing the evolution of disturbances with a prescribed frequency cannot be treated as an initialvalue problem because some eigenvalues of the full system have a negative imaginary part, corresponding to perturbations propagating upstream from the generator (Gill 1965). Reshotko \& Tumin (2001) have addressed this problem by translating the elliptic boundary-value problem into a well-posed initial-value problem spanned only by the first $M$ downstream propagating modes. In this case, the analysis is identical to that carried out by Schmid \& Henningson (1994) for the temporal case. The spatial optimal disturbances are again found to be streamwise elongated vortices and the downstream response corresponds to streamwise streaks.

For the boundary layer, Luchini $(1996,2000)$ and Andersson, Berggren \& Henningson (1999) have overcome the complications inherent in the treatment of the elliptical spatial stability problem by considering the linearized boundary-layer equations instead of the full nonlinear equations. Since their validity is restricted to low-frequency disturbances at high Reynolds numbers, this approach is less general. However, the parabolic nature of the boundary-layer equations allows for the treatment of the spatial streamwise coordinate as a 'time-like' coordinate, for which a simple marching procedure can be employed in the integration. This appears to be very suitable for an application of optimal control theory, which has been used by Luchini (2000) and Andersson et al. (1999) to determine spatial optimal disturbances in boundary layers and by Cathalifaud \& Luchini (2000) to find an optimal control by blowing and suction at the wall.

The objective of optimal control is the determination of an extremum of a suitably specified objective functional which, in the context of flow control, is usually related to 
the kinetic energy of the disturbances. By means of a formal extremization procedure, a system of differential equations (state and adjoint equations) is derived together with optimality conditions. The main advantage of adopting a parabolic model to describe the spatial evolution both of the direct and the adjoint state, is that the solution of the optimality system can be obtained by making use of an iterative optimization algorithm. This procedure permits the determination of the optimal perturbation and/or optimal control functions, starting from arbitrary inflow and wall forcing conditions.

In the present work, we will employ optimal control theory first to find 'worst-case' inflow perturbations in cylindrical pipe flow, and secondly, to determine the best control at the wall, in the form of blowing/suction, capable of counteracting the transient amplification of streaks. To this end we formulate, following Luchini (1996, 2000), a set of linear parabolic equations to describe the spatial evolution of disturbances in cylindrical pipe flow. The use of such an approximation in a study of transient growth and its control, is justified by the observations of Reshotko \& Tumin (2001) that the highest levels of amplification are attained by non-axisymmetric disturbances, that are elongated in the streamwise direction and that vary only slowly with time. The derivation of this linearized parabolic operator for Hagen-Poiseuille flow is considered in $\S 2$.

With the strongest growing disturbances prescribed as inflow conditions, the optimal control, i.e. the wall blowing/suction that hampers the growth of the streaks, can be pursued by following two different strategies: either by seeking a modification of the base flow in order to produce a new base flow less prone to destabilization (Airiau et al. 2003; Zuccher, Luchini \& Bottaro 2004) or by addressing the control efforts directly at diminishing the unstable or algebraically growing disturbances (Bewley \& Liu 1998; Cathalifaud \& Luchini 2000; Corbett \& Bottaro 2001a). In the present work, we will adopt the second technique, also known as cancellation control (Milling 1981; Thomas 1983; Gad-el Hak 2000). Its advantage is that it takes a smaller control energy to efficiently damp infinitesimal perturbations than to modify the base flow. The drawback of cancellation control is that the determined optimally distributed control will be appropriate only for the particular inflow condition provided, which hence must be accurately measured and/or estimated. For instance, the wall blowing/suction distribution in a linear framework must have the right angular phase to be effective. The other technique, i.e. the mean-flow modification, acts independently of such a phase, but it requires a nonlinear model for its optimization.

In summary, our goal is to study, for the case of cylindrical pipe flow, the effect of optimal control on the minimization of the growth of so-called optimal disturbances, i.e. disturbances with maximum amplification under uncontrolled conditions. It is reasonable to argue that a decrease of the disturbance intensity can be interpreted as a spatial delay of the transition process.

After the introduction of the scaling in $\S 2$, we discuss the computation of the optimal disturbances in $\S 3$. Next, we consider in $\S 4$ the formulation of the optimal control together with its results. Finally, in $\S 5$ we briefly consider the case when the worst inflow perturbation is computed simultaneously with the best control at the wall, which is known as robust control (Zhou \& Doyle 1998; Bewley, Temam \& Ziane 2000). This approach takes into account that the presence of the control may possibly change the flow structure and hence the optimal inflow disturbance itself.

\section{Scaling issues and the parabolic model}

We start from the dimensional Navier-Stokes equations in cylindrical coordinates $(\tilde{x}, \tilde{r}, \tilde{\theta})$, with $(\tilde{u}, \tilde{v}, \tilde{w})$ as the corresponding components of the perturbation velocity 
field. After linearization around the laminar solution $\tilde{U} \boldsymbol{e}_{\tilde{x}}$, we introduce a scaling, in which the centreline velocity $\tilde{U}_{0}$ and the radius of the pipe $\tilde{R}$, are taken as the velocity and length scale to non-dimensionalize the axial velocities $\tilde{U}, \tilde{u}$ and the radial coordinate $\tilde{r}$, respectively. Since for the streaky structures that we consider here, variations in the streamwise coordinate are much smaller than those in the radial and tangential direction, $\tilde{x}$ is normalized with a length scale $\tilde{L}$, that satisfies $\tilde{L} \gg \tilde{R}$. From the continuity equation, we can then derive that $\tilde{v}$ and $\tilde{w}$ scale with $\tilde{U}_{0} \tilde{R} / \tilde{L}$. Finally, from the momentum equation, the ratio $\tilde{R} / \tilde{L}$ can be estimated to be of the order of $R e^{-1}$, where for the Reynolds number the standard definition is adopted: $R e=\tilde{U}_{0} \tilde{R} / \nu$, with $v$ the kinematic viscosity. This implies that $\tilde{v}, \tilde{w} \sim R e^{-1} \tilde{U}_{0}$. Time is non-dimensionalized by $\operatorname{Re} \tilde{R} / \tilde{U}_{0}$, which means that this scaling is only valid for low-frequency disturbances $\tilde{\omega} \sim R e^{-1} \tilde{U}_{0} / \tilde{R}$. Finally, the pressure is normalized with $\rho \tilde{U}_{0}^{2} / R e^{2}$, where $\rho$ is the density of the fluid. We note that this scaling for the pressure is appropriate only for non-axisymmetric disturbances because for axisymmetric perturbations a pressure term, constant over the cross-section and of order $\rho \tilde{U}_{0}^{2}$, would have been necessary to account for the confinement of the flow inside a rigid lateral contour. With this in mind and after neglecting terms of relative order $O\left(R e^{-2}\right)$, the following dimensionless equations are obtained:

$$
\begin{gathered}
\frac{\partial v}{\partial t}+U \frac{\partial v}{\partial x}=-\frac{\partial p}{\partial r}+\nabla^{2} v-\frac{v}{r^{2}}-\frac{2}{r^{2}} \frac{\partial w}{\partial \theta}, \\
\frac{\partial u}{\partial t}+U \frac{\partial u}{\partial x}+\frac{\mathrm{d} U}{\mathrm{~d} r} v=\nabla^{2} u, \\
\frac{\partial u}{\partial x}+\frac{1}{r} \frac{\partial(r v)}{\partial r}+\frac{1}{r} \frac{\partial w}{\partial \theta}=0, \\
\frac{\partial w}{\partial t}+U \frac{\partial w}{\partial x}=-\frac{1}{r} \frac{\partial p}{\partial \theta}+\nabla^{2} w-\frac{w}{r^{2}}+\frac{2}{r^{2}} \frac{\partial v}{\partial \theta},
\end{gathered}
$$

with

$$
\nabla^{2}=\frac{1}{r} \frac{\partial}{\partial r}\left(r \frac{\partial}{\partial r}\right)+\frac{1}{r^{2}} \frac{\partial^{2}}{\partial \theta^{2}} .
$$

The Reynolds number does not appear explicitly in these equations, because it has been adopted as a scaling parameter. As a consequence, all the results presented in the following sections are valid for any Reynolds number, provided it is sufficiently large.

Next, we assume for the disturbance quantities $v=(u, v, w)$ and $p$ the following form:

$$
\begin{aligned}
& \boldsymbol{v}(r, \theta, x, t)=\widehat{\boldsymbol{v}}(r, x ; m, \omega) \mathrm{e}^{\mathrm{i}(m \theta-\omega t)}, \\
& p(r, \theta, x, t)=\widehat{p}(r, x ; m, \omega) \mathrm{e}^{\mathrm{i}(m \theta-\omega t)},
\end{aligned}
$$

where $m$ is the azimuthal wavenumber, $\omega$ the dimensionless circular frequency and $\widehat{\boldsymbol{v}}=(\widehat{u}, \widehat{v}, \widehat{w})$ and $\widehat{p}$ the complex disturbance amplitudes. By substituting these expressions into $(2.1 a)-(2.1 d)$ and omitting, from now on, the hat over the variables representing complex amplitudes, we obtain:

$$
\begin{gathered}
-\mathrm{i} \omega v+U \frac{\partial v}{\partial x}=-\frac{\partial p}{\partial r}+\nabla^{2} v-\frac{v}{r^{2}}-\frac{2 \mathrm{i} m}{r^{2}} w \\
-\mathrm{i} \omega u+U \frac{\partial u}{\partial x}+\frac{\mathrm{d} U}{\mathrm{~d} r} v=\nabla^{2} u
\end{gathered}
$$




$$
\begin{gathered}
\frac{\partial u}{\partial x}+\frac{1}{r} \frac{\partial(r v)}{\partial r}+\frac{\mathrm{i} m}{r} w=0, \\
-\mathrm{i} \omega w+U \frac{\partial w}{\partial x}=-\frac{\mathrm{i} m}{r} p+\nabla^{2} w-\frac{w}{r^{2}}+\frac{2 \mathrm{i} m}{r^{2}} v,
\end{gathered}
$$

with

$$
\nabla^{2}=\frac{1}{r} \frac{\partial}{\partial r}\left(r \frac{\partial}{\partial r}\right)-\frac{m^{2}}{r^{2}} .
$$

Equations $(2.3 a)-(2.3 d)$ can be written as a system of first-order differential equations in $x$, for which the vector of the unknowns is given by $\boldsymbol{a}(x, r ; m, \omega)=[v, u, p, w]$. The resulting system in compact notation reads:

$$
\boldsymbol{B} \frac{\partial \boldsymbol{a}}{\partial x}=\boldsymbol{A a}
$$

where $\boldsymbol{A}$ and $\boldsymbol{B}$ are coefficient matrices given by:

$$
\boldsymbol{A}=\left[\begin{array}{cccc}
Q_{1} & 0 & -\frac{\partial}{\partial r} & -\frac{2 \mathrm{i} m}{r^{2}} \\
-U^{\prime} & Q_{2} & 0 & 0 \\
-\frac{1}{r}-\frac{\partial}{\partial r} & 0 & 0 & -\frac{\mathrm{i} m}{r} \\
\frac{2 \mathrm{i} m}{r^{2}} & 0 & -\frac{\mathrm{i} m}{r} & Q_{1}
\end{array}\right], \quad \boldsymbol{B}=\left[\begin{array}{cccc}
U & 0 & 0 & 0 \\
0 & U & 0 & 0 \\
0 & 1 & 0 & 0 \\
0 & 0 & 0 & U
\end{array}\right]
$$

with

$$
Q_{1}=-\frac{\left(m^{2}+1\right)}{r^{2}}+\mathrm{i} \omega+\frac{1}{r} \frac{\partial}{\partial r}+\frac{\partial^{2}}{\partial r^{2}},
$$

and

$$
Q_{2}=-\frac{m^{2}}{r^{2}}+\mathrm{i} \omega+\frac{1}{r} \frac{\partial}{\partial r}+\frac{\partial^{2}}{\partial r^{2}} .
$$

At the pipe wall, the no-slip boundary condition is applied:

$$
r=1: a_{1} \equiv v=0, \quad a_{2} \equiv u=0 \quad a_{4} \equiv w=0 .
$$

At the pipe centreline, the condition is that all flow quantities remain bounded. This is accomplished by the following conditions for $r \rightarrow 0$, as a function of the azimuthal wavenumber $m$ :

$$
\begin{aligned}
& m= \pm 1: a_{1}^{\prime}=a_{2}=a_{4}^{\prime}=0, \Rightarrow a_{3}=0, \\
& |m|>1: a_{1}=a_{2}=a_{4}=0, \Rightarrow a_{3}=0,
\end{aligned}
$$

where a prime indicates a derivative with respect to the radial coordinate.

Equations $(2.3 a)-(2.3 d)$ are Reynolds-independent and parabolic in the streamwise direction. Indeed, the $x$-derivatives in $(2.3 a)-(2.3 d)$ arise from the convective terms in the Navier-Stokes equations rather than from the viscous terms. This enables the integration of (2.5) by means of a marching procedure: given an initial disturbance $\left(u_{0}, v_{0}, w_{0}\right)$ at $x=0$, the downstream development can be obtained by solving an initial boundary-value problem for $x>0$. It must be noted that, because of the nature 
(a)

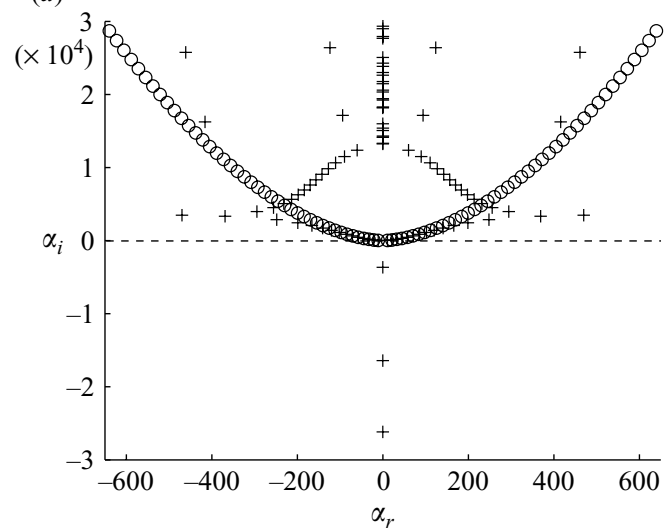

(b)

Figure 1. Spatial spectra of eigenvalues for $m=1: \bigcirc$, parabolic model; + , elliptic system at $R e=3000 .(a): \omega=0,(b): \omega=100, \omega_{e}=0.0333$. The eigenvalues of the elliptic system are multiplied by $R e$.

of $(2.3 a)-(2.3 d)$, only two components of the inflow perturbation velocity can be assigned arbitrarily (see Luchini \& Bottaro 1998). By combining (2.3b) and (2.3c), the following relation can be derived

$$
\left(-r U^{\prime}+U\right) v+r U v^{\prime}+\left(-\frac{m^{2}}{r}+\mathrm{i} \omega r\right) u+u^{\prime}+r u^{\prime \prime}+\mathrm{i} m U w=0,
$$

which must be satisfied by the three velocity components at any $x \geqslant 0$. So to avoid discontinuities in the solution to the initial boundary-value problem at $x=0$, the imposed inflow velocity components $\left(u_{0}, v_{0}, w_{0}\right)$ must satisfy (2.6).

Finally, when the solution of (2.5) is assumed to be of the form:

$$
\boldsymbol{a}(x, r)=\boldsymbol{a}_{\alpha}(r) \mathrm{e}^{\mathrm{i} \alpha x}
$$

the following eigenvalue problem is recovered:

$$
\mathrm{i} \alpha \boldsymbol{B} \boldsymbol{a}_{\alpha}=\boldsymbol{A} \boldsymbol{a}_{\alpha} .
$$

The frequency $\omega$ entering in $\boldsymbol{A}$ is assumed to be real. The spatial stability of the parabolic equations can then be analysed by considering the imaginary part of the complex eigenvalues $\alpha$ of (2.7). These can be related, with the aid of our scaling, to the eigenvalues arising from the complete elliptic linearized Navier-Stokes equations, which are denoted by $\alpha_{e}$. The relation reads: $\alpha=\alpha_{e} R e$. In the unsteady case, the elliptic spectrum must be evaluated for a value of the non-dimensional frequency $\omega_{e}$ satisfying the scaling relation $\omega=\omega_{e} R e$.

The numerical solution of the generalized eigenvalue problem (2.7) is achieved by the use of a Chebyshev pseudospectral collocation technique. Eigenvalues and eigenvectors are computed with the QZ algorithm, implemented in MatLab. The number $N$ of Chebyshev polynomials has been chosen after grid convergence studies in order to ensure well-resolved results for application in the analyses to be performed in the following sections. In figure 1, we show with circles the eigenvalues spectra calculated with $N=128$, for a disturbance with $m=1$, and $\omega=0$ (figure $1 a$ ) and $\omega=100$ (figure $1 b$ ). The elliptic spectra calculated for $R e=3000$ (and for $\omega_{e}=0.0333$ in the unsteady case), are also plotted with crosses, after rescaling with $R e$. For downstream propagating modes the agreement is excellent, as long as $\alpha_{i}$ is sufficiently 
small, also for the case $\omega \neq 0$. Needless to say, the parabolic model does not display any upstream travelling mode, i.e. modes with $\alpha_{i}<0$.

\section{Optimal perturbations}

Spatial optimal disturbances are inflow conditions that excite the largest response from a given base flow. The measure typically used for the size of the disturbance at a specific streamwise location $x$ is its kinetic energy density. Since the streamwise and crossflow velocity components scale differently, the definition of the kinetic energy and its gain to be used in the optimization problem require some attention. In dimensional form, the kinetic energy density in Fourier space is given, apart from a factor $2 \pi$, by:

$$
\tilde{E}(\tilde{x})=\frac{1}{2} \rho \int_{0}^{\tilde{R}}\left(\tilde{u}^{*} \tilde{u}+\tilde{v}^{*} \tilde{v}+\tilde{w}^{*} \tilde{w}\right) \tilde{r} \mathrm{~d} \tilde{r},
$$

where the tilde over the velocity variables denotes here dimensional complex quantities and the asterisk indicates the complex conjugate. When (3.1) is non-dimensionalized with the aid of the scaling described in $\S 2$, the result becomes:

$$
E(x)=\frac{1}{2} \int_{0}^{1}\left[u^{*} u+\operatorname{Re}^{-2}\left(v^{*} v+w^{*} w\right)\right] r \mathrm{~d} r .
$$

We are interested in disturbances of given initial energy $E_{0}$, which undergo significant relative amplification over a given length of the pipe. In other words, our aim is the maximization of the gain $G$ of the system, defined as the ratio between the energy at a final station $x_{\text {fin }}$ and the input energy, i.e.

$$
G\left(x_{f i n}\right)=\frac{E_{x_{f i n}}}{E_{0}}=\frac{\left\{\frac{1}{2} \int_{0}^{1}\left[u^{*} u+R e^{-2}\left(v^{*} v+w^{*} w\right)\right] r \mathrm{~d} r\right\}_{x=x_{f i n}}}{\left\{\frac{1}{2} \int_{0}^{1}\left[u^{*} u+R e^{-2}\left(v^{*} v+w^{*} w\right)\right] r \mathrm{~d} r\right\}_{x=0}} .
$$

As already noted by Luchini (2000) in the context of the boundary-layer approximation, the inflow condition has to be sought among perturbations having $u_{0}=0$, because they lead to a gain of order $R e^{2}$, whereas for $u_{0} \neq 0$ the gain is only of order one. In other words, in the limit $R e \rightarrow \infty$, which is implicit in the parabolic approximation, the gain will always be largest for initial conditions with $u_{0}=0$. On the other hand, an initial perturbation with $u_{0}=0$ produces streaks, for which the streamwise disturbance energy is $R e^{2}$-times larger than the cross-sectional energy. Hence, the disturbance energy at any downstream location $x$ is to leading order the local energy of the streaks, i.e.

$$
E_{u}(x)=\frac{1}{2} \int_{0}^{1} u^{*} u r \mathrm{~d} r
$$

and the maximum gain to leading order is given by:

$$
G\left(x_{f i n}\right)=\frac{E_{u}\left(x_{f i n}\right)}{E_{0}}=\operatorname{Re}^{2} \frac{\left\{\frac{1}{2} \int_{0}^{1} u^{*} u r \mathrm{~d} r\right\}_{x=x_{f i n}}}{\left\{\frac{1}{2} \int_{0}^{1}\left(v^{*} v+w^{*} w\right) r \mathrm{~d} r\right\}_{x=0}},
$$


where the Reynolds number enters as a scaling parameter only, similar to $(2.3 a)-$ $(2.3 d)$.

Although the two definitions (3.3) and (3.5) are equivalent for $R e \rightarrow \infty$, we recover in the latter explicitly the quadratic dependence of the gain $G$ on the Reynolds number. The same relation was achieved in the asymptotic limit of $R e \rightarrow \infty$ by Schmid $\&$ Henningson (1994) by means of a temporal analysis of the elliptic linear operator in Hagen-Poiseuille flow. Throughout the remainder of the paper, we will always rescale the input energy as:

$$
E_{0}=\left\{\frac{1}{2} \int_{0}^{1}\left(v^{*} v+w^{*} w\right) r \mathrm{~d} r\right\}_{x=0}
$$

and, as a consequence, the actual value of the gain is given by $\operatorname{Re}^{2} E_{u}\left(x_{f i n}\right) / E_{0}$.

As mentioned in $\S 1$, the problem of determining the optimal spatial growth as a function of the streamwise coordinate and the associated optimal inflow disturbances can be solved by two different methods. One is the classical technique based on eigenmode expansion introduced by Butler \& Farrell (1992) and extended by Reshotko $\&$ Tumin (2001) to the spatial case. This technique is more straightforward for the determination of global quantities such as the maximum possible energy amplification optimized at any $x$ over all possible inflow perturbations of given energy and it will be used here to check to what extent the parabolic approximation is acceptable. The other technique is based on the mathematical framework of optimal control theory (see Luchini 2000; Cathalifaud \& Luchini 2000; Andersson et al. 1999; Corbett \& Bottaro 2001b) and it will prove very useful in the further determination of the optimal and robust control of 'worst-case' perturbations.

\subsection{Eigenvector expansion}

In the first method, the state vector $\boldsymbol{a}$ is expanded in the first $M$ eigenmodes $\boldsymbol{a}_{\alpha}$ of the eigenvalue problem (2.7), as follows:

$$
\boldsymbol{a}(x, r)=\sum_{k=1}^{M} A_{k} \boldsymbol{a}_{k}(r) \exp \left(\mathrm{i} \alpha_{k} x\right),
$$

where $A_{k}$ is the expansion coefficient of mode $k$. All the modes included in the sum are downstream decaying. Indeed, as can be seen from figure 1, all the eigenvalues of the parabolic spectrum (indicated by circles) have positive imaginary part and consequently the initial-value problem is well-posed.

Next, by introducing a scalar product that, when applied to the state vector with itself, equals the energy density (3.2), the weights of the expansion that maximize the energy growth at any downstream coordinate $x$ for all possible inflow conditions of given energy norm, are determined following the method described by Schmid \& Henningson (1994), which makes use of singular-value decomposition.

For comparison purposes, the maximum gain as a function of $x$ has also been determined for the full elliptic system by restricting the sum in the vector expansion (3.7) to the first $M$ downstream propagating modes (see Reshotko \& Tumin 2001). A number $M=80$ of expansion functions has been used in both cases, while $N=128$ Chebyshev polynomials have been employed for the determination of the eigenfunctions $\boldsymbol{a}_{\alpha}$. The results are shown in figure 2, for $m=1, \omega=0$ and $R e=3000$ in the elliptic case (in the parabolic case, the results are Reynolds-number independent). The azimuthal wavenumber $m=1$ has been considered, since it produces the largest energy amplifications for steady and slightly unsteady perturbations (see Reshotko \& 


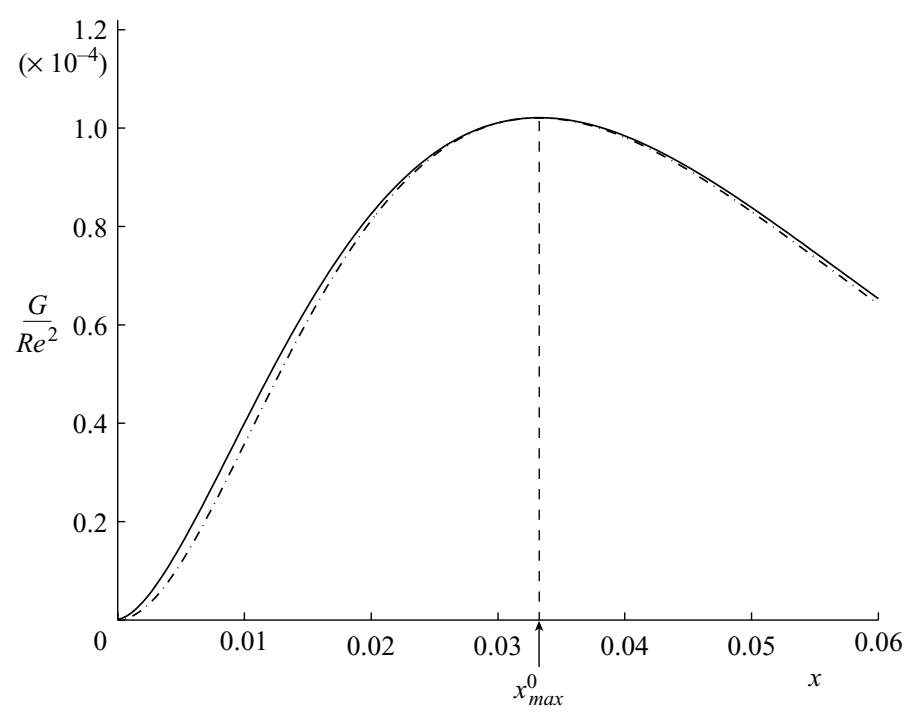

Figure 2. Envelope of the curves of gain versus $x$ for $m=1, \omega=0$ : parabolic system (solid), elliptic system at $R e=3000$ (dashed). The two curves coincide to plotting accuracy. The growth curve for the specific inflow condition that maximizes the energy at $x=x_{\max }^{0}$ is also plotted by the dash-dotted line.

Tumin 2001). Despite the visible differences between the parabolic and elliptic spectra, for stationary disturbances the two models give an identical (i.e. within plotting accuracy) spatial growth curve, even for a reasonably low value of the Reynolds number for the full model. The maximum of the transient growth is achieved in both cases at $x_{\max }^{0}=0.0334$ (the superscript 0 refers to the value of the imposed frequency) in agreement with Reshotko \& Tumin (2001).

The solid line shown in figure 2, represents the outer envelope of the disturbance energy along $x$. It can be thought of as the superposition of several calculations started from different optimal inflow conditions with a given energy norm, specifically targeted for maximum amplification at chosen downstream points. As an example, we show by the dash-dotted line in figure 2, the curve of the energy amplification for the specific inflow condition that maximizes the gain at $x=x_{\max }^{0}$. This curve has been computed with the second technique, based on optimal control theory, which is discussed further below. For this particular solution we show in figure 3(a), the inflow disturbance, which consists of a pair of counter-rotating vortices, with negligible, i.e. to first-order zero, axial velocity. The result is exactly the same for the elliptic or the parabolic model. Owing to the lift-up mechanism, i.e. the advection of mean shear by the two vortices, this inflow disturbance yields further downstream at $x=x_{\max }^{0}$ strong streaks of high and low longitudinal velocity as shown in figure $3(b)$.

In figure 4, the transient growth curves are shown for the case of unsteady perturbations. In figure $4(a)$, we present the curves computed with the parabolic model for $\omega=100$ and $\omega=300$, and with the elliptic model. For the latter, the curves are computed for $R e=3000$ so that $\omega_{e}$ is equal to 0.0333 and 0.1 , respectively, in order to satisfy the scaling relation $\omega=\omega_{e} R e$. The difference in the results obtained with both models is small. In figure $4(b)$, the same comparison is shown for $\omega=750$ and for two different values of the Reynolds number. As expected, for this larger value of $\omega$, leading to a higher $\omega_{e}$ at the value $R e=3000$, the result for the gain $G$ obtained from the parabolic model has deteriorated in comparison with the result of 

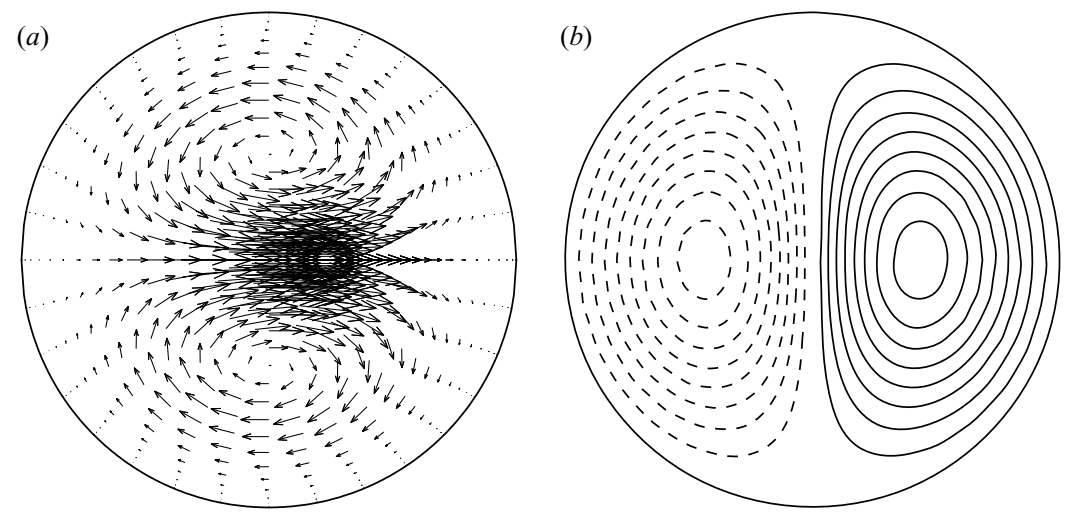

FIGURE 3. (a) Vector field in the $(r, \theta)$ plane, of the optimal perturbation with $m=1, \omega=0$, that maximizes the gain $G$ at $x=x_{\text {max }}^{0}$. (b) Contour plot of the resulting streaks at $x=x_{\max }^{0}$ : continuous and dashed lines are used for positive and negative values respectively.

(a)

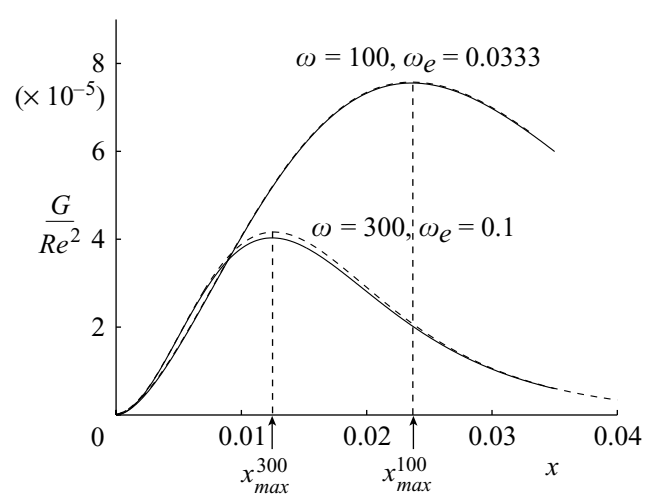

(b)

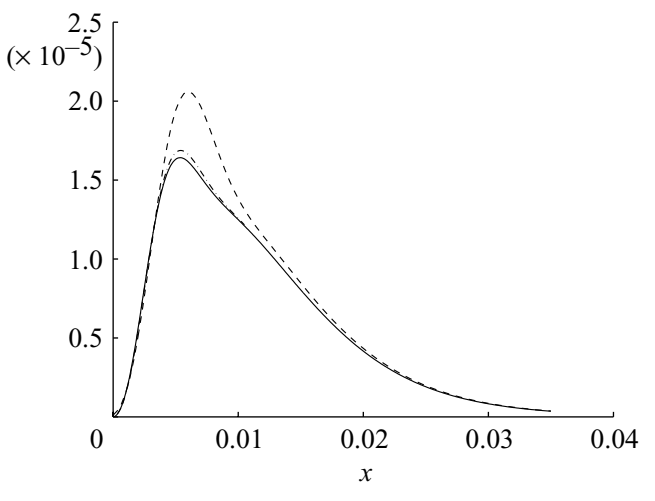

FIgURE 4. Transient growth as a function of the streamwise coordinate for $m=1$. (a) Parabolic system (solid), elliptic system at $R e=3000$ (dashed). (b) Parabolic system at $\omega=750$ (solid), elliptic system at $R e=3000, \omega_{e}=0.25$ (dashed) and at $R e=10^{4}, \omega_{e}=0.075$ (dash-dotted).

the elliptic model. Figure $4(b)$ also shows that the agreement improves for the larger value of the Reynolds number considered, i.e. $R e=10^{4}$, implying a lower value of $\omega_{e}$. However, in studies dealing with the onset and delay of transition in HagenPoiseuille flow, the Reynolds numbers of interest lie primarily around 3000, for which the parabolic approximation underestimates the maximum attainable growth by more than $3 \%$ when $\omega>300$.

The inflow disturbance that produces the largest energy amplification at $\omega=100$ is plotted in figure 5 together with the resulting streaks at $x_{\max }^{100}$, which is the position where the maximum gain is achieved. The flow field is rather different from that observed in the steady case, cf. figure 3 . It oscillates sinusoidally in the cross-section around the solution shown in the figure. For $\omega=300$, the disturbance flow field is qualitatively the same.

\subsection{Direct and adjoint systems}

The second technique, which we have mentioned above as a method for determining the inflow perturbation that yields the highest energy amplification at a fixed 
(a)

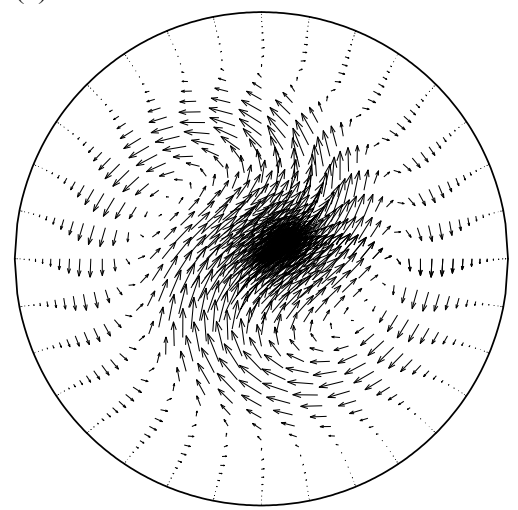

(b)

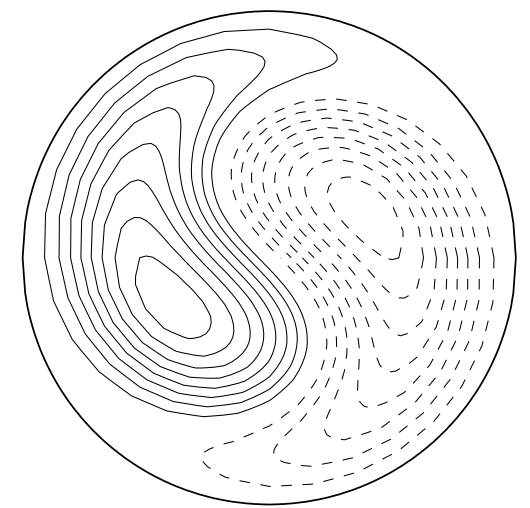

Figure 5. (a) Vector field in the $(r, \theta)$ plane, of the optimal perturbation with $m=1, \omega=100$, that maximizes the gain $\mathrm{G}$ at $x=x_{\max }^{100}$. $(b)$ Contour plot of the resulting streaks at $x=x_{\max }^{100}$.

downstream position, makes use of optimal control theory. The essential elements that have to be defined in this method are: (i) a mathematical description of the objective to be attained expressed in the form of a cost functional $\mathscr{I}$; (ii) some means of adjusting the system to meet the objective; and (iii) a description in terms of constraints of the system and its behaviour (see, for instance, Abergel \& Temam 1990; Gunzburger 1997). In our specific problem, the objective is the maximization of the growth experienced by the perturbation, therefore the cost functional is defined as:

$$
\mathscr{I}=\frac{G\left(x_{f i n}\right)}{\operatorname{Re}^{2}}=\frac{E_{u}\left(x_{f i n}\right)}{E_{0}} .
$$

The control, in this case, is the inflow condition itself $\left(0, v_{0}, w_{0}\right)$, since it directly affects the state of the system. As mentioned in $\S 2$, only one of the two non-zero velocity components can be updated independently, say $v_{0}$, and the other can be derived from (2.6), with $u \equiv 0$ and $v=v_{0}$. It is useful to introduce an explicit statement that the state at $x=0$ matches the 'control':

$$
\mathscr{H}_{0}\left(v(0, r), v_{0}(r)\right)=v(0, r)-v_{0}(r)=0 .
$$

Finally, the constraints are the equations and the boundary conditions that describe the evolution of the system at any position $x$. In the following, we will consider the parabolic system (2.5) with its boundary conditions. The method adopted in this paper to solve the optimization problem is based on Lagrange multipliers (see, for instance, Gunzburger 1997). According to this method, the constrained maximization problem is reduced to an unconstrained one, by introducing the adjoint variables $\boldsymbol{b}(x, r)=\left(b_{1}, b_{2}, b_{3}, b_{4}\right)$ and $c_{0}(r)$ to satisfy the constraints and the control statement (3.9). These play the role of Lagrange multipliers in the augmented functional $\mathscr{L}$ :

$$
\mathscr{L}\left(\boldsymbol{a}, v_{0}, \boldsymbol{b}, c_{0}\right)=\mathscr{I}-\left\langle\left[\boldsymbol{B} \frac{\partial \boldsymbol{a}}{\partial x}-\boldsymbol{A} \boldsymbol{a}\right], \boldsymbol{b}\right\rangle-\left(\mathscr{H}_{0}, c_{0}\right),
$$

where the inner products are defined by:

$$
\langle\boldsymbol{p}, \boldsymbol{q}\rangle=\int_{0}^{x_{f i n}} \int_{0}^{1} \boldsymbol{q}^{\dagger} \boldsymbol{p} r \mathrm{~d} r \mathrm{~d} x+\text { c.c. }
$$


and by

$$
(\boldsymbol{p}, \boldsymbol{q})=\int_{0}^{1} \boldsymbol{q}^{\dagger} \boldsymbol{p} r \mathrm{~d} r+\text { c.c. }
$$

Here, the $\dagger$ denotes the transpose complex conjugate and c.c. the complex conjugate of the first term in the expression. The addition of this complex conjugate is required to ensure that the Lagrangian functional $\mathscr{L}$ is real. In the definition of $\mathscr{L}$ we have not explicitly enforced the constraints given by the boundary conditions, since they are not subject to variations. They must nonetheless be satisfied by the solution to the optimal control problem.

Each argument of the functional $\mathscr{L}$ is considered to be an independent variable and the optimal control problem consists now in determining the inflow velocity $v_{0}$, the state $\boldsymbol{a}$ and the adjoint state $\left(\boldsymbol{b}, c_{0}\right)$, which render $\mathscr{L}$ stationary. A necessary condition for an extremum point of $\mathscr{L}$ is that its variations with respect to each of its arguments, expressed by Fréchet derivatives in the corresponding directions, vanish. Hence, the variation of the functional that must be forced to zero, reads:

$$
\delta \mathscr{L}=\frac{\partial \mathscr{L}}{\partial \boldsymbol{a}} \delta \boldsymbol{a}+\frac{\partial \mathscr{L}}{\partial v_{0}} \delta v_{0}+\frac{\partial \mathscr{L}}{\partial \boldsymbol{b}} \delta \boldsymbol{b}+\frac{\partial \mathscr{L}}{\partial c_{0}} \delta c_{0} .
$$

Upon setting the first variation of the Lagrangian with respect to the adjoint variables to zero, i.e. $(\partial \mathscr{L} / \partial \boldsymbol{b}) \delta \boldsymbol{b}=0$ and $\left(\partial \mathscr{L} / \partial c_{0}\right) \delta c_{0}=0$, we recover the original constraints (2.5) and (3.9). By forcing the first variation of the Lagrangian with respect to the state variables to zero, i.e. $(\partial \mathscr{L} / \partial \boldsymbol{a}) \delta \boldsymbol{a}=0$, we obtain the following adjoint equations:

$$
\begin{gathered}
U \frac{\partial b_{1}}{\partial x}=\left[\frac{\left(m^{2}+1\right)}{r^{2}}+\mathrm{i} \omega\right] b_{1}-\frac{1}{r} b_{1}^{\prime}-b_{1}^{\prime \prime}+U^{\prime} b_{2}+\frac{1}{r^{2}} b_{3}-\frac{1}{r} b_{3}^{\prime}+\frac{2 \mathrm{i} m}{r^{2}} b_{4}, \\
U \frac{\partial b_{2}}{\partial x}+\frac{1}{r} \frac{\partial b_{3}}{\partial x}=\left(\frac{m^{2}}{r^{2}}+\mathrm{i} \omega r^{2}\right) b_{2}-\frac{1}{r} b_{2}^{\prime}-b_{2}^{\prime \prime}+b_{0}, \\
\frac{1}{r} b_{1}+b_{1}^{\prime}+\frac{\mathrm{i} m}{r} b_{4}=0, \\
U \frac{\partial b_{4}}{\partial x}=-\frac{2 \mathrm{i} m}{r^{2}} b_{1}-\frac{\mathrm{i} m}{r^{2}} b_{3}+\left[\frac{\left(m^{2}+1\right)}{r^{2}}+\mathrm{i} \omega\right] b_{4}-\frac{1}{r} b_{4}^{\prime}-b_{4}^{\prime \prime},
\end{gathered}
$$

where $b_{0}$ is an inhomogeneous term, which vanishes in this case, but which will turn out to be useful when we consider control. The adjoint system is parabolic in the backward direction, which is the only direction of stable evolution for the adjoint state. Therefore, initial (sometimes also called 'terminal') conditions at $x=x_{\text {fin }}$ are required. These are provided by the process of integration by parts, from which the adjoint equations are obtained, and they are given by:

$$
\begin{aligned}
U b_{1}\left(x_{f i n}, r\right) & =0, \\
U b_{2}\left(x_{f i n}, r\right)+\frac{b_{3}\left(x_{f i n}, r\right)}{r} & =\frac{1}{2 E_{0}} u\left(x_{f i n}, r\right), \\
U b_{4}\left(x_{f i n}, r\right) & =0 .
\end{aligned}
$$

In addition from the condition $(\partial \mathscr{L} / \partial v) \delta v=0$, it follows that:

$$
U b_{1}(0, r)=c_{0}(r)
$$


and upon setting $\left(\partial \mathscr{L} / \partial v_{0}\right) \delta v_{0}$ to zero, the following optimality condition is recovered:

$$
\frac{E_{u}\left(x_{f i n}\right)}{2 E_{0}^{2}} v_{0}=c_{0}(r) \text {. }
$$

By combining (3.14) and (3.15), an expression for the inflow perturbation as a function of the adjoint variable $b_{1}$ at $x=0$, can be derived:

$$
v_{0}(r)=U b_{1}(0, r) \frac{2 E_{0}^{2}}{E_{u}\left(x_{f i n}\right)} .
$$

By substituting $u \equiv 0$ and $v=v_{0}$ in (2.6) and by using (3.12c), it is found that $w_{0}=2 U b_{4}(0, r) E_{0}^{2} / E_{u}\left(x_{f i n}\right)$. Hence, the adjoint variables $b_{1}(0, r)$ and $b_{4}(0, r)$ represent receptivity functions that provide the response of the system to forcing at the inflow.

The optimal perturbation now satisfies the system that consists of the direct equations (2.5) and their boundary conditions, the adjoint system $(3.12 a)-(3.12 d)$ and the optimality condition (3.16). Moreover, it has to maximize the cost function (3.8). Numerically, the solution is reached with the help of an iterative algorithm that involves successive integrations of the direct and adjoint equations. Starting from an arbitrary estimate for $v_{0}$ (and $w_{0}$ ), (2.5) is integrated forwards in the axial direction $x$ up to a prescribed final position $x_{f i n}$. Then $(3.13 a)-(3.13 c)$ are used to determine terminal conditions for the adjoint equations, which are subsequently integrated backwards in $x$ up to $x=0$. With the help of (3.16), we then find a new estimate for the optimal perturbation, which is used to update the inflow disturbance in the course of the iterations. At the end of each forward integration, a convergence test on the objective functional is performed. If $\left|\mathscr{I}^{(n)}-\mathscr{I}^{(n-1)}\right| / \mathscr{I}^{(n)}<10^{-12}$, where the superscript indicates the iteration number, the case is considered converged.

It must be noted that this approach might at first seem to yield only a local extremum. The numerical results show, however, that a global maximum of $\mathscr{I}$ is found within a few iterations, with the optimal $v_{0}$ satisfying the imposed requirements. This is related to the fact that the procedure adopted is equivalent to performing power iterations to isolate the largest singular value of the operator propagating the initial condition downstream. Provided the initial guess at $x=0$ is not orthogonal to the first singular vector, it is easy to verify that power iterations will always converge to the first singular value, i.e. to the maximum gain.

The numerical solution of the optimality system is achieved with the aid of a Chebyshev pseudospectral collocation technique for the discretization along the radial direction and a second-order backward Euler finite-difference scheme for the integration in the axial direction. The same methods are employed for the integration both of the direct and the adjoint equations. To check the correct implementation of this method, we have plotted in figure 2 as a dash-dotted line the growth curve of the optimal steady perturbation that maximizes the energy at $x=x_{\max }^{0}$. A number $N=128$ of Chebyshev polynomials and a grid spacing $\mathrm{d} x=10^{-4}$ have been used for these calculations. As can be seen, the growth curve is tangent to the envelope curve, calculated with the first technique, at the position $x=x_{\max }^{0}$. Moreover, the results for the optimal perturbation are found to be identical to those shown in figure 3 .

\section{Optimal control}

We now turn to the determination of the optimal control, via blowing/suction at the wall, of the elongated streaks that result from the amplification of the optimal perturbation. Hence, a worst-case disturbance is imposed at the inflow pipe section 
and its evolution in $x$ is described by the state vector $\boldsymbol{a}$, constrained by (2.5) and related boundary conditions. Upon normalization of the inflow disturbance such that its energy $E_{0}$ is equal to one, the intensity of the perturbation at any downstream location $x$ is given, to leading order, by $E_{u}(x)$ and the actual value of the gain by $R^{2} E_{u}(x)$. Control is applied over the interval $x \in\left[x_{i n}, x_{f i n}\right]$, where $x_{i n}$ and $x_{f i n}$ can be arbitrarily varied. Along this distance we prescribe the radial velocity at the wall to be equal to the control function $v_{w}(x)$, where for the control velocity the same dependence in $\theta$ and $\omega$ is assumed as for the disturbance field. It is useful to introduce an explicit statement for the control:

$$
\mathscr{H}_{c}\left(v, v_{w}\right)=v(x, 1)-v_{w}(x)=0 .
$$

The magnitude of the control can be quantified by the control energy density defined as:

$$
E_{c}=\frac{1}{2} \int_{x_{i n}}^{x_{f i n}} v_{w}^{*} v_{w} \mathrm{~d} x
$$

The natural choice for the control's objective is to minimize the energy at the end of the control interval, provided that at the same time the energy required to control the system also remains small. However, the use of a cost function including only the two quantities described above, can induce a larger initial increase of perturbation kinetic energy than that experienced in the uncontrolled case (see Cathalifaud \& Luchini 2000). This can be avoided by adding a term to the cost function, which is proportional to the average energy of perturbations in the control interval. Therefore we will make use of the following definition of the (real) cost functional (see Corbett \& Bottaro 2001a):

$$
\mathscr{I}\left(\boldsymbol{v}, v_{w}\right)=\zeta E_{c}+\chi E_{u}\left(x_{f i n}\right)+\psi \int_{x_{i n}}^{x_{f i n}} E_{u}(x) \mathrm{d} x .
$$

The real constants $\zeta, \chi$ and $\psi$ are weighting parameters that can be adjusted to penalize a contribution to the cost, through which one can choose among different control strategies. The optimal control problem consists of the determination of a control $v_{w}$ and a state $\boldsymbol{a}$ which minimize the cost function (4.3), subject to the constraint that state and control satisfy (2.5) and its boundary conditions. This constrained minimization problem can be reduced to the unconstrained problem of determining an extremum for the augmented Lagrangian functional $\mathscr{L}$, defined as:

$$
\mathscr{L}\left(\boldsymbol{a}, v_{w}, \boldsymbol{b}, c\right)=\mathscr{I}-\left\langle\boldsymbol{B} \frac{\partial \boldsymbol{a}}{\partial x}-\boldsymbol{A} \boldsymbol{a}, \boldsymbol{b}\right\rangle-\left[\mathscr{H}_{c}, c\right],
$$

where $\boldsymbol{b}$ and $c$ are the Lagrange multipliers which enforce (2.5) and (4.1). As in the constrained case, a necessary condition for an extremum point is that the first variation of $\mathscr{L}$ with respect to each of its arguments vanishes. Enforcing the condition $(\partial \mathscr{L} / \partial \boldsymbol{a}) \delta \boldsymbol{a}=0$ leads to the adjoint equations $(3.12 a)-(3.12 d)$ with the only difference that the forcing term $b_{0}=-\psi u / 2$ appears on the right-hand side of $(3.12 b)$.

Boundary and terminal conditions for the adjoint system are found, like in $\S 3$, as a result of the process of integration by parts. These are the same as those found in $\S 3$ except for the right-hand side of equation (3.13b) which is here equal to $\chi u\left(x_{f i n}, r\right) / 2$. In addition, from $(\partial \mathscr{L} / \partial v) \delta v=0$ it is found:

$$
c(x)=-b_{3}(x, 1) .
$$


Enforcing stationarity with respect to the control, i.e. $\left(\partial \mathscr{L} / \partial v_{w}\right) \delta v_{w}=0$, yields the optimality condition:

$$
\frac{1}{2} \zeta v_{w}(x)=-c(x)
$$

Together with (4.5), this expression leads to the following result for the optimal control:

$$
v_{w}(x)=\frac{1}{2} \zeta b_{3}(x, 1),
$$

linking the optimal normal velocity at the wall to the adjoint variable $b_{3}(x, 1)$. The latter is the Green's function defining the receptivity of the flow to radial velocity disturbances at the wall (Luchini \& Bottaro 1998). The optimality system that must be satisfied by the optimal control solution comprises the direct equations (2.5), with a fixed value for the perturbation at $x=0$, namely the optimal disturbance, the adjoint system $(3.12 a)-(3.12 d)$ and the condition (4.7) for the control.

To solve this system, an iterative algorithm is used similar to that of the previous section: starting from an arbitrary estimate for $v_{w},(2.5)$ is integrated forwards in the streamwise coordinate $x$ up to $x=x_{\text {fin }}$, while the axial velocity $u$ is stored at each step. Then $(3.13 a)-(3.13 c)$ is used to determine terminal conditions for $(3.12 a)-(3.12 d)$, which are subsequently integrated backwards in $x$ up to the initial controlled position $x=x_{i n}$. Along the way, the value of the adjoint variable $b_{3}$ at the wall is retained. A new estimate for the control can be obtained by imposing:

$$
v_{w}^{(n+1)}=v_{w}^{(n)}-\beta\left[v_{w}^{(n)}-\frac{2}{\zeta} b_{3}(x, 1)\right]^{(n)},
$$

where $\beta$ is a relaxation parameter. The expression in parentheses represents, up to a multiplicative factor, the gradient of the cost function $\mathscr{I}$ with respect to the control $v_{w}$. When $\beta$ is chosen to be a constant or to minimize the cost at each iteration, a simple gradient or a steepest descent method are, respectively, employed. However, the convergence rate of these two techniques, especially of the first one, is rather low. A faster convergence towards the optimal solution can be obtained by employing the Polack-Ribiere variant of the standard conjugate gradient method (Press et al. 1992), a technique already used successfully by Corbett \& Bottaro (2001a) to solve a similar problem in the boundary layer. Like the steepest-descent method, this approach requires one line minimization per iteration, implying a number of forward integrations of (2.5).

\subsection{Results}

For the computations reported here, we employ 65 collocation points in the radial direction and a constant step equal to $\mathrm{d} x=10^{-4}$ in the axial direction. Grid convergence studies show that these settings are adequate to ensure well-resolved results.

The control is applied to the optimal perturbations as inflow condition, i.e. perturbations experiencing the largest algebraic growth. The control interval is chosen to extend from the inflow section up to the position where a peak in disturbance energy is observed for the uncontrolled reference case. Hence, in (4.3) $x_{i n}=0$ and $x_{f i n}=x_{\max }^{\omega}$. The integration of the direct equations is, however, extended beyond $x_{f i n}$ in order to assess the behaviour of the system after control is turned off.

As already mentioned, it is possible to adopt different control strategies by varying the weighting parameters in the definition of the cost function. Following the terminology introduced by Corbett \& Bottaro (2001a), the control strategy corresponding 


\begin{tabular}{|c|c|c|c|c|c|c|c|c|c|}
\hline$\omega$ & $x_{\max }^{\omega}$ & Strategy & $\zeta$ & $\chi$ & $\psi$ & $\mathscr{I}$ & $E_{c}$ & $E_{u}\left(x_{f i n}\right)$ & $E_{u} \mathrm{~d} x$ \\
\hline \multirow[t]{4}{*}{0} & 0.0334 & equil & 1 & $2 \times 10^{2}$ & $6 \times 10^{3}$ & 0.0058 & 0.00192 & $8.8356 \times 10^{-6}$ & $3.5570 \times 10^{-7}$ \\
\hline & & cheap & 5 & $2 \times 10^{2}$ & $6 \times 10^{3}$ & 0.0115 & 0.00108 & $1.6354 \times 10^{-5}$ & $4.7215 \times 10^{-7}$ \\
\hline & & flat & 1 & $2 \times 10^{2}$ & $6 \times 10^{4}$ & 0.0247 & 0.00188 & $9.5462 \times 10^{-6}$ & $3.4861 \times 10^{-7}$ \\
\hline & & small & 1 & $2 \times 10^{3}$ & $6 \times 10^{3}$ & 0.0151 & 0.00386 & $3.5799 \times 10^{-6}$ & $6.8158 \times 10^{-7}$ \\
\hline \multirow[t]{4}{*}{100} & 0.0235 & equil & 0.5 & $2 \times 10^{2}$ & $6 \times 10^{3}$ & 0.00436 & 0.002267 & $9.4893 \times 10^{-6}$ & $2.2165 \times 10^{-7}$ \\
\hline & & cheap & 5 & $2 \times 10^{2}$ & $6 \times 10^{3}$ & 0.00979 & $7.3276 \times 10^{-4}$ & $2.0711 \times 10^{-5}$ & $3.3083 \times 10^{-7}$ \\
\hline & & flat & 0.5 & $2 \times 10^{2}$ & $6 \times 10^{4}$ & 0.0156 & 0.00203 & $1.1252 \times 10^{-5}$ & $2.0546 \times 10^{-7}$ \\
\hline & & small & 0.5 & $2 \times 10^{3}$ & $6 \times 10^{3}$ & 0.0141 & 0.00606 & $3.7563 \times 10^{-6}$ & $5.9156 \times 10^{-7}$ \\
\hline \multirow[t]{4}{*}{300} & 0.013 & equil & 0.5 & $2 \times 10^{2}$ & $6 \times 10^{3}$ & 0.00336 & 0.002089 & $9.2282 \times 10^{-6}$ & $7.7916 \times 10^{-8}$ \\
\hline & & cheap & 5 & $2 \times 10^{2}$ & $6 \times 10^{3}$ & 0.00651 & $2.9737 \times 10^{-4}$ & $2.0548 \times 10^{-5}$ & $1.5173 \times 10^{-7}$ \\
\hline & & flat & 0.5 & $2 \times 10^{2}$ & $6 \times 10^{4}$ & 0.006945 & 0.002468 & $9.4317 \times 10^{-6}$ & $6.3739 \times 10^{-8}$ \\
\hline & & small & 0.5 & $2 \times 10^{3}$ & $6 \times 10^{3}$ & 0.01321 & 0.008067 & $4.0991 \times 10^{-6}$ & $1.6263 \times 10^{-7}$ \\
\hline
\end{tabular}

TABLE 1. Parameter settings, value of the cost functional, control and perturbation energies for different control strategies of optimal perturbations, acting on the interval $\left[0, x_{\max }^{\omega}\right]$.
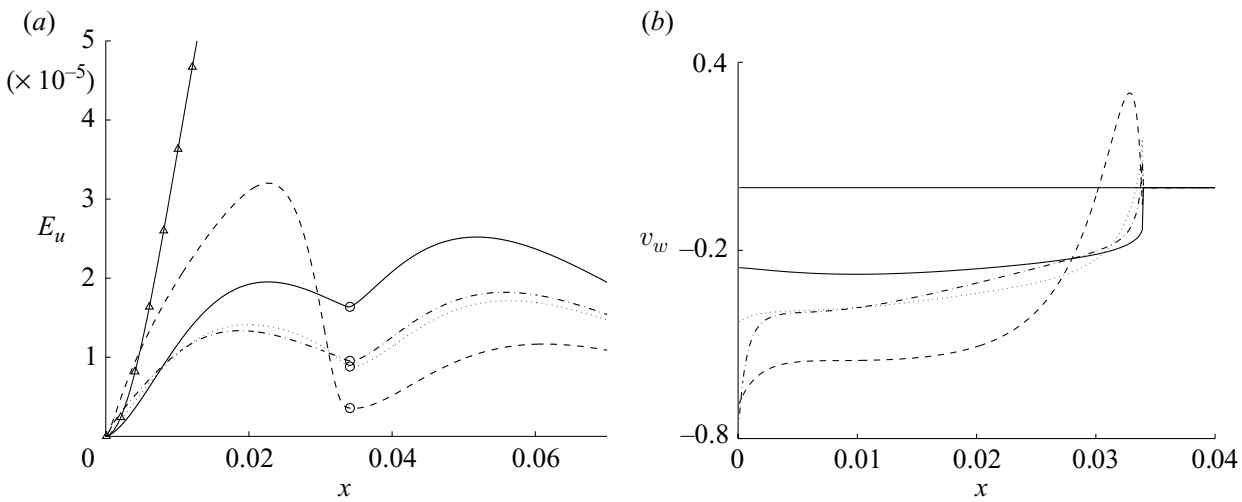

FIGURE 6. (a) Growth curves in the presence of different control strategies, with circles indicating the end of the control interval $x=x_{f i n}$, and $(b)$ corresponding wall velocity distributions, for $m=1, \omega=0$ : 'equil' (dotted); 'cheap' (solid); 'flat' (dash-dotted); 'small' (dashed); uncontrolled case (solid with triangles). For all the strategies, the control function has zero imaginary part.

to the value of the parameters for which the contribution of each term in (4.3) is of the same order of magnitude is termed 'equil', for 'equilibrium control'. Starting from these reference values and increasing only the value of $\zeta$, implies penalization of the control energy, leading to a strategy termed 'cheap' since a small control effort is imposed. An increase in $\chi$ produces a control which targets mostly the final energy of the streaks, and this strategy is termed 'small', while setting a higher value for $\psi$ penalizes initial excursions in the disturbance energy, which hence attains a rather uniform distribution along the $x$-direction: this strategy is thus called 'flat'. The values of the parameters adopted for each strategy are given in table 1.

The resulting growth curves and optimal distributions of blowing/suction at the wall are given in figure 6 for the steady case, and in figures 7 and 8 for $\omega=100$ and $\omega=300$, respectively. The curve of the energy amplification in the uncontrolled case is also shown for comparison. 

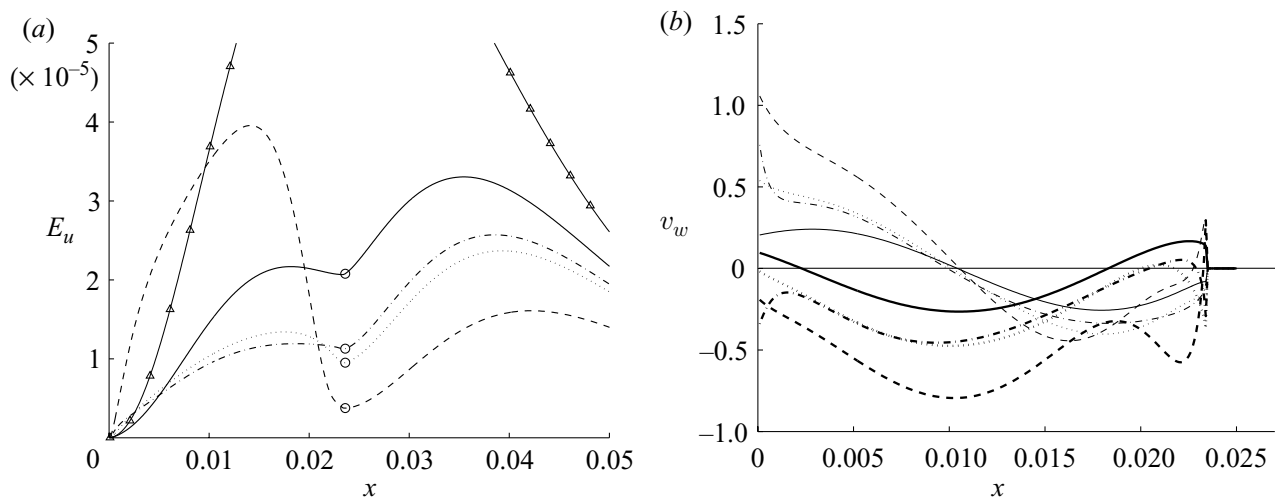

FIgURE 7. As figure 6 , but $\omega=100$. In $(b)$, thick and thin lines are used, respectively, for the real and imaginary parts of the control function.
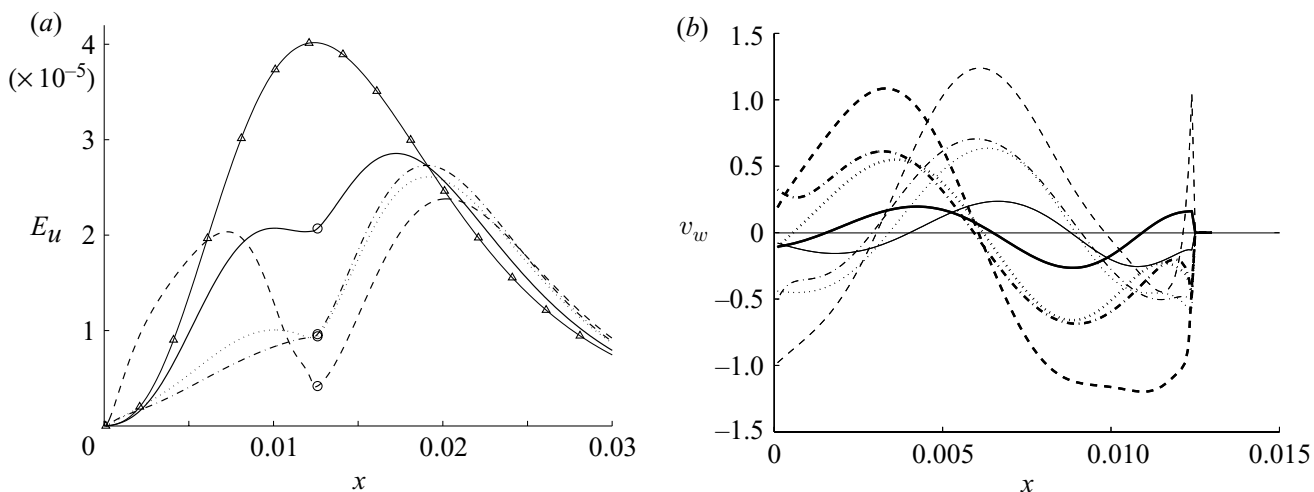

FIGURE 8. As figure 7, but $\omega=300$.

In the steady case, the radial and azimuthal velocity components of the optimal inflow perturbation and the resulting streaks, have constant phases that differ by $90^{\circ}$. Since the angular orientation of the two vortices is arbitrary, the inflow disturbance and the control are configured, without loss of generality, in such a way that only the real part of the control function is different from zero (see figure 6b).

In all the cases analysed, every one of the strategies proposed shows some degree of effectiveness in attenuating the disturbance over the control window. As mentioned in $\S 1$, the effectiveness of the control depends strongly on the angular alignment of the radial velocity at the wall with the inflow perturbation. For instance, in the steady case, the cost $\mathscr{I}$ as a function of the difference of the angular phases of the control and the optimal disturbance shows a minimum value for a phase difference equal to $\pi$ (as found for the optimally determined control functions). Moreover, a deviation from this value causes strong increases in the cost $\mathscr{I}$ that can become even larger than in the uncontrolled case. Although this result is straightforward, it is useful to emphasize the importance of properly sensing the initial condition in order to apply an appropriate control. It will also be useful in $\S 4.3$, where we will discuss the ability of linearly derived optimal control laws to treat some nonlinear cases.

The energy growth curves plotted in figures 6, 7 and 8, show that downstream of the control region, amplification by transient growth appears again. This growth is more 

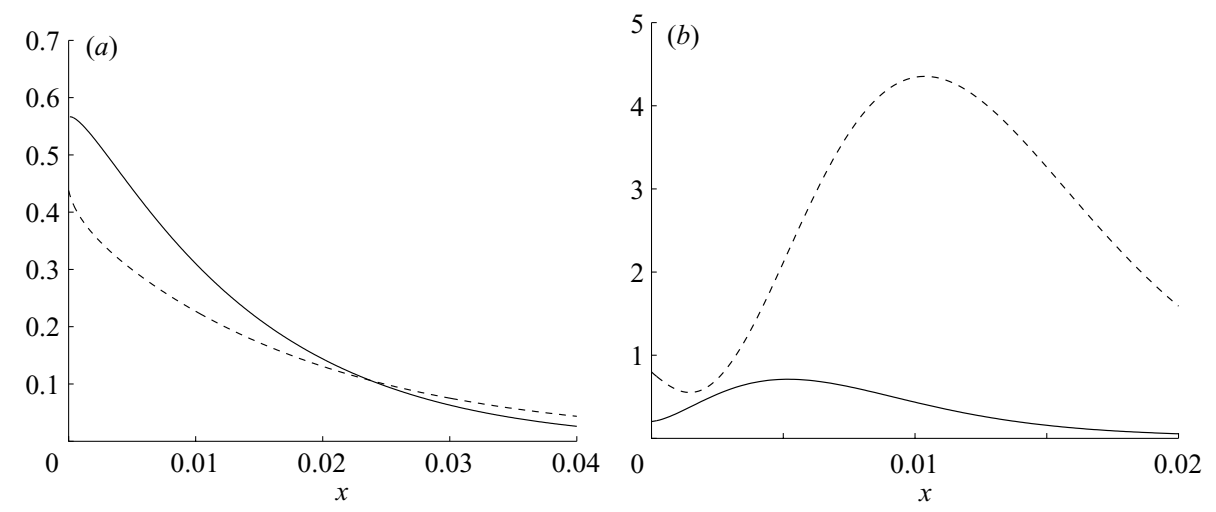

FIGURE 9. Radial (solid line) and azimuthal (dashed line) kinetic energy densities as functions of the streamwise coordinate for optimal inflow perturbations with $m=1$ and $(a) \omega=0$, (b) $\omega=300$.

pronounced for those strategies that perform poorly in damping the energy at $x=x_{f i n}$, this is particularly the case for unsteady perturbations that appear to be more difficult to control. This is possibly related to the fact that in the unsteady case the energy growth is not only due to the amplification of the streamwise disturbance component, but also to that of the cross-flow components. In figure 9, the kinetic energy density associated to the radial and azimuthal disturbance velocity components is plotted as a function of the streamwise coordinate, for optimal inflow perturbations with $(a)$ $\omega=0$ and $(b) \omega=300$. For steady perturbations, all the amplification is related to the streamwise component while the other components are seen to decay from their inflow values. For unsteady perturbations, the amplification is also primarily related to the streamwise component, but the other components, especially the azimuthal one, also undergo an amplification from their inflow values. This behaviour, the counterpart of which in the temporal setting was pointed out by Bergström (1993), could be responsible for the diminished effectiveness of our boundary control.

The 'cheap' strategy is the least effective in terms of the achieved energy reduction over the whole domain. It follows, however, from table 1 that in the steady case, the control effort for this strategy amounts to only about half of that employed for the 'equil' and 'flat' strategies and to about a quarter of that employed in the 'small' strategy. In the unsteady cases shown in figures 7 and 8 , the effort connected to the 'cheap' strategy is even lower compared to the other ones. Moreover, the control distribution is very smooth and almost constant over the whole control length, a characteristic that renders this strategy appealing when envisaging practical applications.

In the 'small' strategy, the energy at the end of the control interval is by definition the smallest compared to all other control strategies. The post-control amplification seems also to be the smallest, but a closer inspection reveals that the growth rate of the energy for this strategy is the largest at $x=x_{f i n}$. Still, the smaller value of the energy at this final point guarantees that the energy resulting from the growth remains lower than in the other cases. The wall forcing connected to the 'small' strategy leads to the largest disturbance energy within the controlled region. Initially, the growth is even higher than the transient growth that we seek to eliminate. Therefore, we conclude that the energy spent in this control strategy, which is the largest of all strategies employed, is not efficiently used. 
In the 'flat' control strategy, which penalizes mostly the term in the cost functional related to the integral of the disturbance energy over the whole control domain, the disturbance energy is forced to a low and almost constant level over the whole control interval. This is achieved at the expense of a rather high amount of control effort that is mostly concentrated in the first part of the optimization interval to rapidly bring the disturbance energy to a low value. This results in a sharp peak (also present to a lesser extent in the 'small' strategy) in the initial distribution of the radial velocity at the wall.

The presence of steep gradients in the control function, that appear for certain settings of the parameters $\zeta, \chi, \psi$ in the cost functional $\mathscr{I}$, could contradict the hypothesis of slow streamwise variation of the disturbance flow field, that is at the basis of the parabolic model governing its evolution. These peaks could have been avoided $a$ priori by introducing a term in the cost function (4.3) that limits the axial derivative of the control velocity over the optimization interval. This has been done, for example, by Airiau et al. (2003) in a related work on optimal steady suction for transition delay in a boundary layer, where the same assumption of slow streamwise variation was adopted. The appropriateness of the parabolic model in representing flows with rapid variations in the radial velocity at the wall can also be verified $a$ posteriori, by comparing the numerical results achieved without any penalization of the gradient of the control function, with those obtained from the complete NavierStokes equations, i.e. without the parabolic approximation, for a pipe flow subject to the control laws derived from the parabolic model. Some comparisons will be discussed in $\S 4.3$.

The physical mechanism responsible for the observed reduction in perturbation kinetic energy can be inferred by studying the evolution of the disturbance under active control. For instance, in figure 10 we show the disturbance for the steady case, at different downstream positions under the action of 'flat' control. The optimal perturbation at the inflow consists of a pair of counter-rotating vortices with a negligible $u$-velocity component. The wall blowing/suction leads to the confinement of these two vortices within a circular region of smaller radius. Hence, they are displaced further away from the wall, i.e. in a region where the gradient of the mean velocity is smaller. As a consequence, the effect of the lift-up mechanism that is responsible for transient growth is reduced. Moreover, two half-vortices, created at the wall by the control itself, induce 'buffer streaks' in the near-wall region which suppress the principal streaks generated by the optimal perturbation. The persistence of lowamplitude streamwise vortices downstream of the controlled region, as observable in figure $10(f)$, produces again weak streaks. Thus, the transient growth connected to these vortices is responsible for the post-control amplification in the energy growth curves, observable in figure $6(a)$.

In figure 11, the development of the optimal steady perturbation under the action of the different control strategies, is shown at the cross-section $x=x_{f i n} / 2$. The primary difference amongst the control strategies lies in the intensity of the buffer streaks, that appears to be proportional to the magnitude of the control. The 'flat' and 'small' strategies displace the principal streaks further away from the wall than, for example, the 'cheap' strategy, and this translates into a diminished effectiveness of the lift-up mechanism.

\subsection{Control on a finite window}

In all the cases analysed up to now, control has always been applied from the inflow section up to the axial position where the optimal perturbation experiences its largest 
(a)

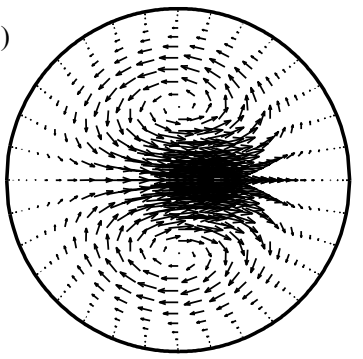

(c)

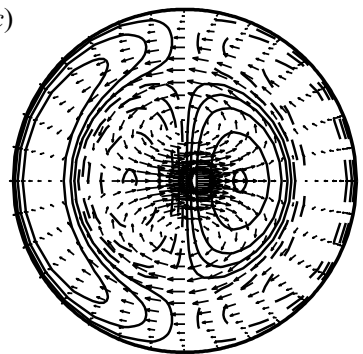

(e)

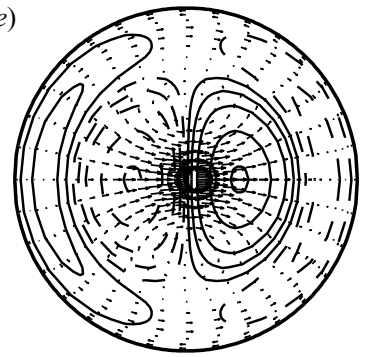

(b)

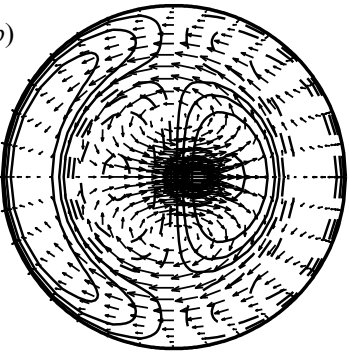

(d)

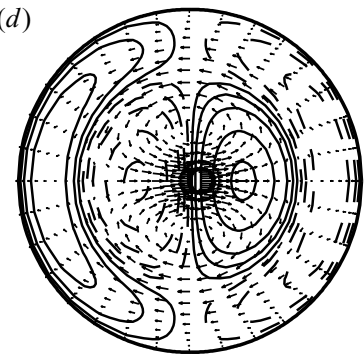

$(f)$

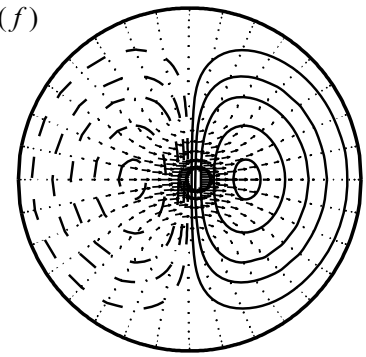

FIGURE 10. Spatial evolution of the optimal steady disturbance subject to the 'flat' control strategy: (a) $x=0,(b) x=x_{f i n} / 4,(c) x=x_{f i n} / 2,(d) x=3 / 4 x_{f i n},(e) x=x_{f i n},(f) x=5 / 4 x_{f i n}$. Shown are contours of $u$ (dashed lines are negative) and vector plots of the cross-flow velocity components. Contour levels and vector scaling are identical in all cases.

energy growth in the uncontrolled case. However, this control distance can amount to several pipe diameters. Such a long length of control could be impossible to implement in real applications. In this context, it would be more practical to envisage that blowing/suction at the wall is applied along a limited length or over a number of short consecutive portions of the pipe. The question is whether it is possible to achieve the same order of reduction in perturbation kinetic energy than with the continuous control analysed above. This question can be answered by considering a case, in which the control in the cost functional (4.3) is penalized by a hat-shaped function or a smoothed-hat weight function $S(x)$. For the latter we use:

$$
S(x)= \begin{cases}1-\left[\sin \left(\pi \frac{x-x_{1}}{x_{2}-x_{1}}-\frac{1}{2} \pi\right)\right]^{20} & \text { for } x_{1}<x<x_{2}, \\ 0 & \text { for } x \leqslant x_{1} \text { and } x \geqslant x_{2}\end{cases}
$$

where $x_{1}$ and $x_{2}$ are the edges of the window where control is active.

In figures 12 and 13 the results obtained with the limited control length and with the steady optimal disturbance as inflow condition are displayed. The parameters 

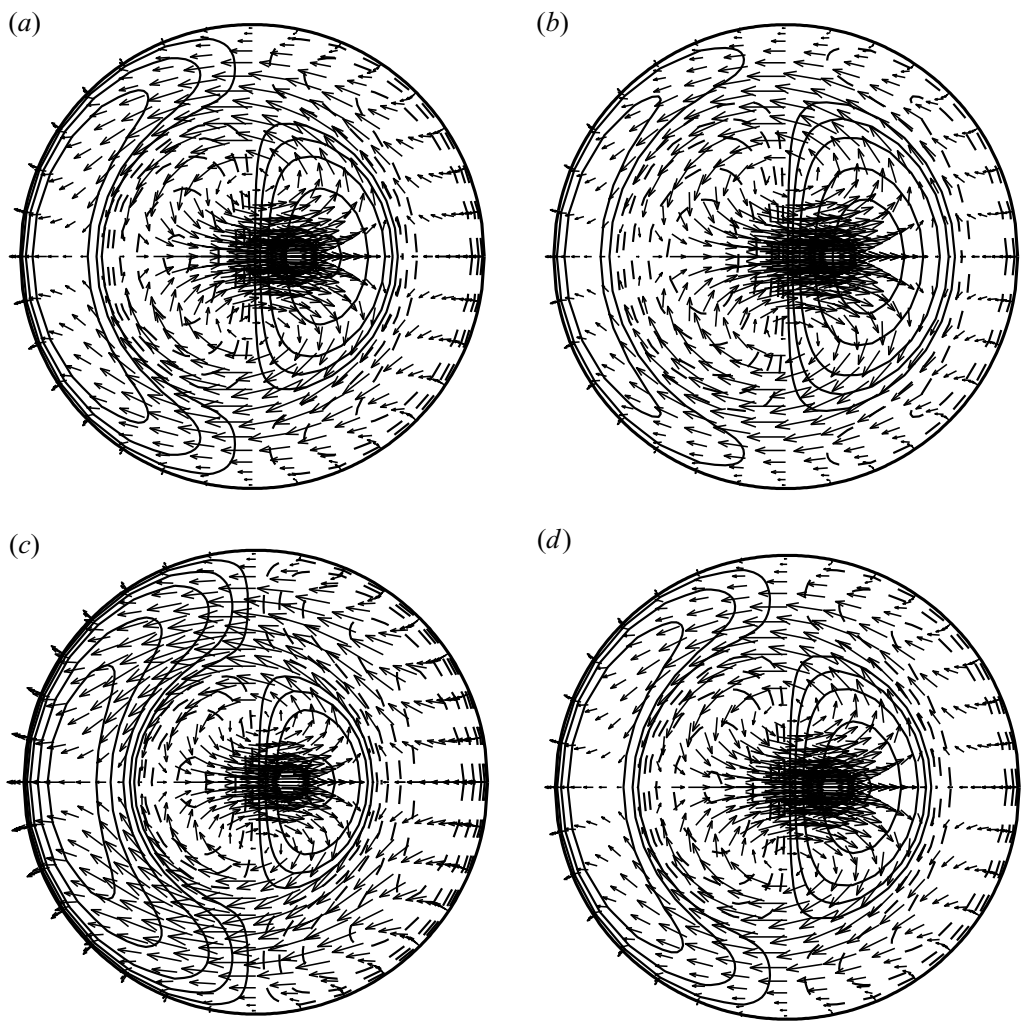

FiguRE 11. Optimal steady disturbance at $x=x_{f i n} / 2$, subject to various control strategies: $(a)$ 'equil', $(b)$ 'cheap', $(c)$ 'small', $(d)$ 'flat'. Contour levels and vector scaling are identical in all cases.
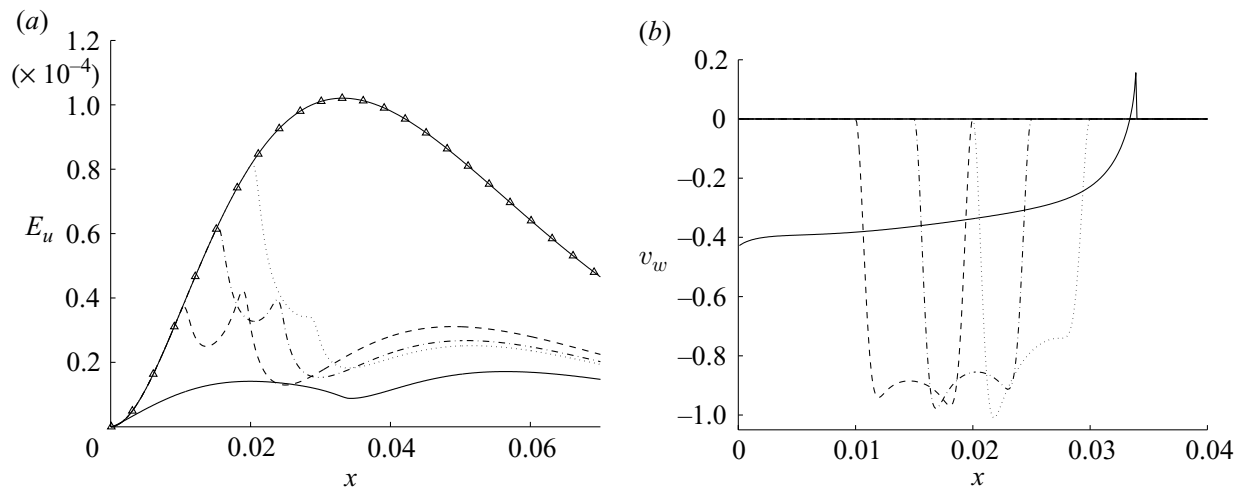

FIGURE 12. (a) Growth curves and (b) corresponding wall velocity distributions, for $m=1$, $\omega=0$, in the uncontrolled case (solid line with triangles), with the parameter settings $\zeta=1$, $\chi=200, \psi=6000$, and for different control windows: $x \in\left[0, x_{\max }^{0}\right]$ (solid), $x \in[0.01,0.02]$ (dashed), $x \in[0.015,0.025]$ (dash-dotted), $x \in[0.02,0.03]$ (dotted).

adopted in the cost function (4.3) correspond to the 'equil' control strategy. The growth curves in the uncontrolled case and with control applied from $x=0$ up to $x=x_{\max }^{0}$ are also included in these figures for reference. In figure 12, we consider different configurations with control applied over a single window of fixed width 

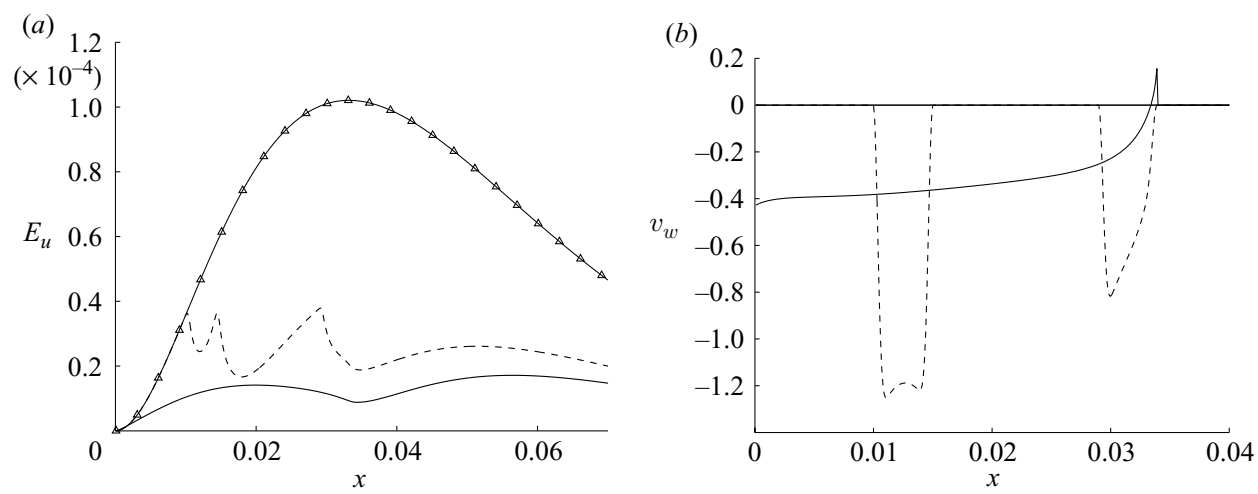

FIGURE 13. As for figure 12, but for control windows: $x \in\left[0, x_{\max }^{0}\right]$ (solid), $x \in[0.01,0.015] \cup\left[0.029, x_{\max }^{0}\right]$ (dashed).

\begin{tabular}{ccccc}
{$\left[x_{\text {in }} x_{\text {fin }}\right]$} & \multicolumn{1}{c}{} & \multicolumn{1}{c}{$E_{c}$} & $E_{u}\left(x_{\text {fin }}\right)$ & $\int E_{u} \mathrm{~d} x$ \\
{$[0.010 .02]$} & & & & \\
{$[0.0150 .025]$} & 0.01201 & 0.00392 & $2.226 \times 10^{-5}$ & $6.934 \times 10^{-7}$ \\
{$[0.020 .03]$} & 0.01242 & 0.00325 & $1.772 \times 10^{-5}$ & $9.369 \times 10^{-7}$ \\
{$[0.010 .015]+\left[0.029 x_{\text {max }}^{0}\right]$} & 0.0123 & 0.003925 & $1.9087 \times 10^{-5}$ & $7.6317 \times 10^{-7}$
\end{tabular}

TABLE 2. 'Equil' control strategy of steady perturbations with $m=1$. Given are: control window, value of the cost functional, control and perturbation energies.

$\Delta=0.01$, but with the window positioned at different streamwise locations. For all the cases analysed, an overall reduction of perturbation kinetic energy is observed, which is about equal to the reduction obtained in the case with continuous control between $\left[x_{i n}, x_{f i n}\right]$. However, from numerical results given in table 2 it can be inferred that the position of the control window, for which the lowest value of $\mathscr{I}$ is achieved, is the most upstream one.

For the results shown in figure 13, control is applied over two separate shorter windows of equal width $\Delta=0.005$. The first window is positioned close to the inflow section where, from the results discussed above, it appears that control has a large effect. The second window is placed further downstream to counteract the post-control amplification behind the first window. This configuration achieves a significant reduction in perturbation kinetic energy over the whole domain, although the control effort is approximately twice as large as in the continuous optimal control configuration.

\subsubsection{Optimal window position}

From figure 12, it appears that for the position of the control window that attains the lowest value of $\mathscr{I}$ (i.e. the most upstream one, among the positions considered), the peak values of the control function $v_{w}(x)$ near the edges of the window are about equal. Here, we will show that this is exactly the condition that must be satisfied by the control function when, for any given value of $\Delta$, the distance of the leading edge of the control window from the inflow section, say $x_{w}^{o p t}$, is introduced as an additional optimization variable. The problem is the minimization of a weighted sum of the final energy at $x=x_{\max }^{0}$, the energy averaged over the whole interval $\left[0, x_{\max }^{0}\right]$ and the control energy, with wall forcing only locally active over a finite length $\Delta$ along the 
pipe. For simplicity, we penalize the control function by a hat-shaped function, such that $v(x, 1)=v_{w}(x)$ for $x \in\left[x_{w}^{o p t}, x_{w}^{o p t}+\Delta\right]$ and $v(x, 1)=0$ elsewhere. Thus, the control effort entering in (4.3) is quantified by:

$$
E_{c}=\frac{1}{2} \int_{x_{w}^{o p t}}^{x_{w}^{o p t}+\Delta}\left(v_{w}^{*} v_{w}\right) \mathrm{d} x
$$

and the Lagrangian function reads:

$$
\mathscr{L}\left(\boldsymbol{a}, v_{w}, \boldsymbol{b}, c, x_{w}^{o p t}\right)=\mathscr{I}-\left\langle\boldsymbol{B} \frac{\partial \boldsymbol{a}}{\partial x}-\boldsymbol{A} \boldsymbol{a}, \boldsymbol{b}\right\rangle-\int_{x_{w}^{o p t}}^{x_{w}^{o p t}+\Delta} c^{*}\left[v(x, 1)-v_{w}(x)\right] \mathrm{d} x .
$$

Setting the first variation of $\mathscr{L}$ with respect to each of its arguments to zero, leads to the same adjoint equations, terminal and optimality conditions as derived above for the optimal control applied over the whole distance of growth of the algebraically unstable perturbations. Additionally, by making use of the Leibniz theorem, the first variation of $\mathscr{L}$ with respect to $x_{w}^{o p t}$, is given by:

$$
\begin{aligned}
\frac{\partial \mathscr{L}}{\partial x_{w}^{o p t}}=\frac{1}{2} \zeta\left[\left.\left(v_{w}^{*} v_{w}\right)\right|_{x_{w}^{o p t}+\Delta}-\left.\left(v_{w}^{*} v_{w}\right)\right|_{x_{w}^{o p t}}\right] & -\left.c^{*}\left(x_{w}^{o p t}+\Delta\right)\left[v(x, 1)-v_{w}(x)\right]\right|_{x_{w}^{o p t}+\Delta} \\
& +\left.c^{*}\left(x_{w}^{o p t}\right)\left[v(x, 1)-v_{w}(x)\right]\right|_{x_{w}^{o p t}}
\end{aligned}
$$

Since, according to our constraint, the radial velocity at the wall is equal to the control velocity at the edges of the finite window, the last two terms in (4.11) are identically zero. Therefore, by forcing $\partial \mathscr{L} / \partial x_{w}^{o p t}$ to zero, it is easy to derive that the optimal position of the control strip is the one for which:

$$
\left|v_{w}\left(x_{w}^{o p t}+\Delta\right)\right|=\left|v_{w}\left(x_{w}^{o p t}\right)\right| \text {. }
$$

\subsection{Navier-Stokes results}

Full simulations of the controlled flows considered above are performed with the help of a direct-simulation code developed by Eggels et al. (1994). This code computes the flow in a cylindrical pipe geometry by a finite-volume technique with secondorder accuracy for the spatial discretization. The code has already been applied by Gavarini, Bottaro \& Nieuwstadt (2004) in order to simulate the spatial evolution of disturbances to the base flow and the ensuing transition to turbulence. At the inflow boundary, an optimal perturbation is imposed with a prescribed amplitude, whereas at the outflow, a fringe region is implemented to gradually damp the disturbances flowing out of the physical domain with minimal reflection. For further details, we refer to Eggels et al. (1994) and Gavarini et al. (2004).

A first set of computations is carried out to check the appropriateness of the parabolic model in representing flows with rapid variations in the radial velocity at the wall. Then calculations are performed to test the effectiveness of the optimal wall blowing/suction distributions calculated by means of linear theory, in controlling perturbations of finite amplitude. Both series of simulations are carried out at a Reynolds number of interest for transition, i.e. $R e=3000$. The computational domain in the streamwise direction consists of a physical distance that measures $\left(\operatorname{Re} x_{\max }^{\omega}\right)$ radii, plus the fringe region. In the figures that follow, only the physical domain is shown. An acceptable resolution is achieved by employing $64 \times 32$ grid points in the radial and azimuthal directions and a uniform grid spacing with $\Delta \tilde{x} / \tilde{R}=0.1875$ in the axial direction.

The relevance of the parabolic model is evaluated both for steady and for unsteady $(\omega=300)$ inflow disturbances. In each case, the optimal perturbation is imposed at the inflow section with an amplitude $A_{v}$ equal to $R e^{-1}$, whereas in the interval 

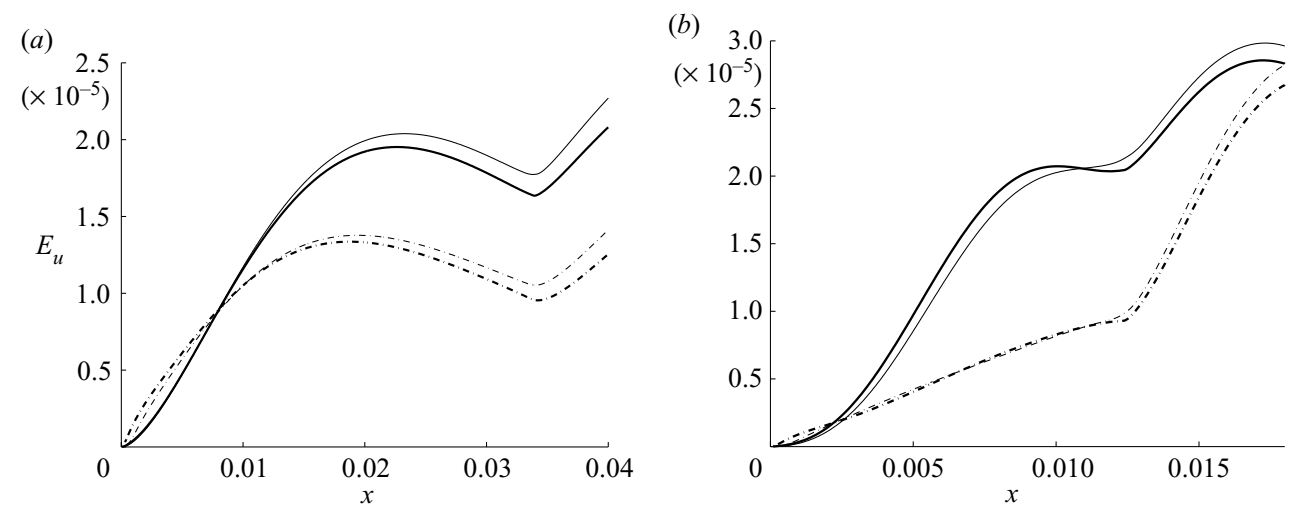

FiguRE 14. Transient growth curves for $m=1,(a) \omega=0$ and $(b) \omega=300$ or $\omega_{e}=0.1$, under the action of control: comparison between the parabolic approach (thick lines) and Navier-Stokes computations for $R e=3000$ (thin lines). Solid lines, 'cheap' strategy; dash-dotted lines, 'flat' strategy.
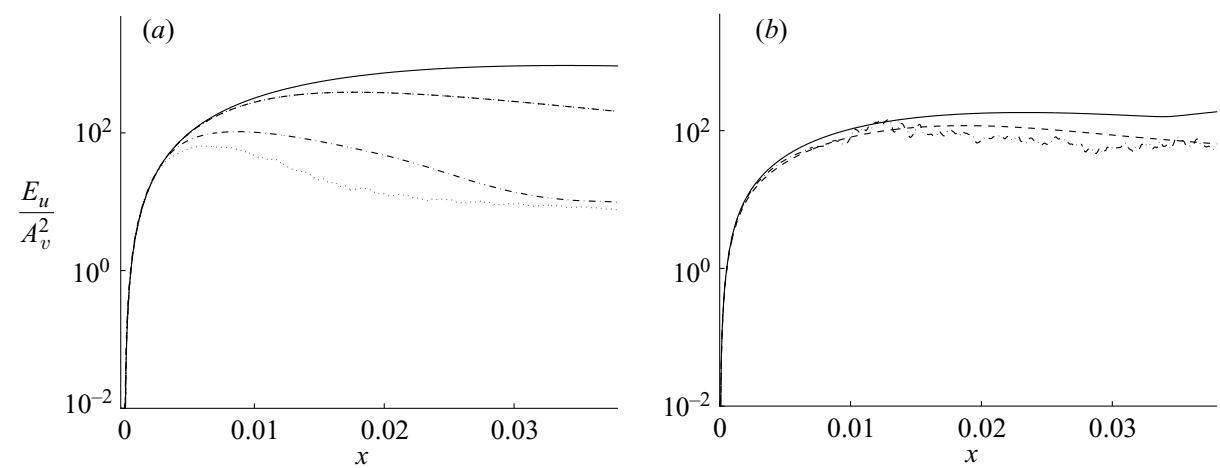

FIGURE 15. Streamwise kinetic energy density scaled by the inflow amplitude squared, as a function of the axial coordinate. The inflow condition is given by the optimal perturbation with $m=1, \omega=0$ and $A_{v}=R e^{-1}$ (solid), $A_{v}=0.01$ (dashed), $A_{v}=0.02$ (dash-dotted), $A_{v}=0.04$ (dotted). (a) Uncontrolled case, $(b)$ 'cheap' control applied over the interval $\left[0, x_{\max }^{0}\right]$.

$\tilde{x} / \tilde{R} \in\left[0, \operatorname{Re}_{\text {max }}^{\omega}\right]$ an inhomogeneous boundary condition is used for the radial velocity component. This is set equal to the optimal control functions $v_{w}(x)$, as obtained in the previous sections and shown in figures $6(b)$ and $8(b)$, scaled by the Reynolds number.

In figure 14, a comparison is given between the growth curves obtained with the parabolic and the full models, for the 'cheap' and 'flat' control strategies. As can be seen, the agreement is very good both for the steady and the unsteady cases, even for the 'flat' strategy, for which we have shown that the control function has a steep gradient close to the inflow section. This means that the parabolic model adopted in this paper is able to predict with sufficient accuracy the evolution of the state of the system, in the presence of the calculated optimal wall blowing/suction distributions.

The effectiveness of the control in damping finite-amplitude perturbations is then assessed by performing a series of simulations where the steady optimal disturbance is imposed as the inflow condition with increasing amplitude values. The resulting streamwise distribution of the perturbation kinetic energy $E_{u}$ of the streaks, normalized by the inflow amplitude squared, is shown in figure 15. Results are presented for the simulations carried out both for the uncontrolled flow (figure 15a) 

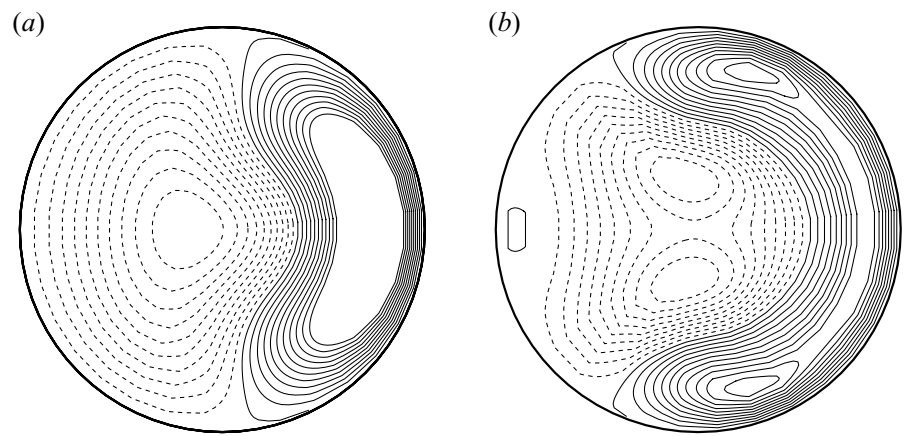

FIGURE 16. Contour plot of the streamwise disturbance velocity at $x=0.0133$ : continuous and dashed lines are used for positive and negative values, respectively. The inflow condition is given by the optimal perturbation with $m=1, \omega=0$ and $(a) A_{v}=0.01,(b) A_{v}=0.02$. Contour values from -0.38 to 0.4 with spacing equal to 0.039 in $(a)$, and from -0.46 to 0.52 with spacing equal to 0.049 in $(b)$.

and for the flow field subject to the 'cheap' control strategy (figure 15b). In this case, the results are obtained by first multiplying the control function $v_{w}(x)$ by $A_{v}$ and then applying this as an inhomogeneous boundary condition in the interval $\left[0, \operatorname{Re} x_{\max }^{0}\right]$.

In the uncontrolled case, transition is observed for the largest-amplitude value considered, i.e. $A_{v}=0.04$, when a random perturbation equal to $1 \%$ of $A_{v}$ is added to the inflow disturbance to break its streamwise symmetry. The $A_{v}=R e^{-1}$ curve corresponds to the linear evolution, whereas for increasing values of the inflow amplitude the nonlinearities result clearly in a saturation of the perturbation energy. A departure from the linear behaviour is already visible for $A_{v}=0.01$. The effect of the nonlinearities can also be recognized by looking at the $u$ velocity component at a downstream cross-section. In figure 16, for instance, this is shown at $x=0.0133$ for two values of $A_{v}$ in the nonlinear regime, namely $A_{v}=0.01$ and $A_{v}=0.02$. As the inflow amplitude increases, the flow structure is more and more distorted compared to the linear case where it is dominated by regularly distributed streaks resembling those shown in figure $3(b)$.

The results presented in figure 15(b) show that when 'cheap' control is applied, an overall reduction of perturbation kinetic energy is achieved for the lower-amplitude values, i.e. $A_{v}=R e^{-1}$ and $A_{v}=0.01$. Instead, for $A_{v}=0.02$, the control works properly only up to $x \simeq 0.01$, corresponding to the position of maximum energy in the uncontrolled flow, but further downstream it induces an early transition. Recalling the importance of the alignment of the control with respect to the perturbations, the effect observed is presumably due to the different structure of the flow field in the presence of relatively high-amplitude disturbances as compared to the linear evolution. For $A_{v}=0.01$, figure 16 shows that the flow field is not too distorted compared to the linear case and control still works, whereas for $A_{v}=0.02$ the secondary motion is substantially different and the applied control even becomes detrimental. We conclude that for large initial amplitudes full nonlinear calculations of the optimal control laws become indispensable (Zuccher et al. 2004).

\section{Robust control}

In the previous section, we have computed the optimal velocity distribution at the wall that minimizes the algebraic growth of a given inflow perturbation, which is chosen to be the one undergoing the largest energy amplification in an uncontrolled 
flow. However, the presence of the control possibly changes the 'worst' inflow condition. It is thus necessary that this is recomputed simultaneously with the 'best' control at the wall. For this approach, known as robust control (Bewley et al. 2000), we use a slightly different definition of the cost functional (4.3) given by:

$$
\mathscr{I}\left(\boldsymbol{v}, v_{w}\right)=\chi E_{u}\left(x_{f i n}\right)+\psi \int_{x_{i n}}^{x_{f i n}} E_{u}(x) \mathrm{d} x .
$$

Here, the energy of the control is not incorporated in the cost functional, but it is imposed as an additional constraint, in the form:

$$
E_{c}\left(v_{w}\right)=E_{c_{0}},
$$

where $E_{c_{0}}$ is a given value. When this is taken to be equal to the energy of the optimal control distributions calculated for the various strategies in the previous section, a direct comparison with the optimal control results is obtained. The problem of robust control can now be formulated as the simultaneous determination of the inflow perturbation that maximizes the cost functional (5.1) and the wall-normal velocity that minimizes it. This non-cooperative strategy thus implies that we are looking for a saddle-point solution of (5.1). The inflow condition is an additional unknown and its energy must also be constrained, as follows:

$$
E_{0}\left(v_{0}\right)=1 .
$$

The inflow energy is written as a functional of $v_{0}$ only, since $w_{0}$ is related to $v_{0}$ by (2.6) and it is normalized to 1 to compare with the optimal control results. The optimization procedure is analogous to that adopted for the determination of the worst inflow disturbances and the best control separately. The only difference is that now they are to be computed simultaneously. This implies that we are looking for a saddle-point solution of (5.1). The Lagrangian functional is given in this case by:

$$
\begin{aligned}
\mathscr{L}\left(\boldsymbol{a}, v_{0}, v_{w}, \boldsymbol{b}, c_{0}, c, \lambda_{0}, \lambda_{w}\right)=\mathscr{I}- & \left\langle\boldsymbol{B} \frac{\partial \boldsymbol{a}}{\partial x}-\boldsymbol{A} \boldsymbol{a}, \boldsymbol{b}\right\rangle-\left[\mathscr{H}_{c}, c\right]-\left[\mathscr{H}_{0}, c_{0}\right] \\
& -\lambda_{w}\left(E_{c}\left(v_{w}\right)-E_{c_{0}}\right)-\lambda_{0}\left(E_{0}\left(v_{0}\right)-1\right),
\end{aligned}
$$

where $\lambda_{w}$ and $\lambda_{0}$ are two new Lagrange multipliers for the constraints (5.2) and (5.3). Setting the Fréchet derivative of $\mathscr{L}$ with respect to the state $\boldsymbol{a}$ equal to zero, leads to the same adjoint equations and terminal conditions as those derived for the optimal control problem. Moreover, $(\partial \mathscr{L} / \partial v) \delta v=0$ yields the two relations (3.14) and (4.5). Finally, by enforcing stationarity with respect to the inflow velocity and to the control, i.e. $\left(\partial \mathscr{L} / \partial v_{0}\right) \delta v_{0}=0$ and $\left(\partial \mathscr{L} / \partial v_{w}\right) \delta v_{w}=0$, the following optimality conditions are obtained:

$$
c_{0}=\frac{1}{2} \lambda_{0} v_{0}, \quad c=\frac{1}{2} \lambda_{w} v_{w} .
$$

These, together with (3.14) and (4.5), lead to the following expressions for the perturbation at $x=0$ and for the wall boundary condition:

$$
v_{0}(r)=\frac{2 U b_{1}(0, r)}{\lambda_{0}}, \quad v_{w}(x)=\frac{-2 b_{3}(x, 1)}{\lambda_{w}},
$$


where $\lambda_{0}$ and $\lambda_{w}$ are given by:

$$
\lambda_{0}= \pm \sqrt{2 \int_{0}^{1} U^{2}\left(b_{1}^{*} b_{1}+b_{4}^{*} b_{4}\right) r \mathrm{~d} r}, \quad \lambda_{w}= \pm \sqrt{\frac{2 \int_{x_{i n}}^{x_{f i n}}\left(b_{3}^{*} b_{3}\right) \mathrm{d} x}{E_{w}}} .
$$

The optimality system that must be satisfied by the robust solution comprises the direct and adjoint equations (2.5) and $(3.12 a)-(3.12 d)$ with their boundary conditions, and the optimality conditions (5.6). Moreover, the solution must coincide with a saddle point in parameter space, corresponding to a maximum of $\mathscr{I}$ with respect to $v_{0}$ and to a minimum relative to $v_{w}$.

An iterative technique, first proposed by Bewley et al. (2000), similar to that already outlined for the solution of the optimal-perturbation and optimal-control problems of the previous sections, is employed to solve the optimality system. Unlike the case for the two separate analyses, where only one of the two optimality conditions (5.6) has to be updated, in the present problem we must update each condition in turn. More specifically, the optimal perturbation is calculated first, following the procedure outlined in $\S 3.2$, i.e. by performing a series of ascent iterations until convergence to the optimal solution, for an arbitrary given wall forcing with fixed energy $E_{c_{0}}$. Then, the optimal control of this inflow disturbance is determined, as outlined in $\S 4$, by performing a number of descent iterations. At this point, convergence towards the saddle-point solution becomes more critical, and we have found it necessary to perform one iteration of the optimal-perturbation problem followed by one iteration of the optimal-control problem. In addition, for those strategies in which the control effort is higher and the wall forcing distribution is less smooth, namely the 'small' and the 'flat' strategies, it was necessary to adopt a relaxation factor in the update of the optimality condition for the inflow disturbance. Finally, a bisection search method is used whenever in the iteration for the maximization of $\mathscr{I}$ a lower value of the cost function is found.

A typical convergence curve, showing the cost function against the iteration number for a representative case is displayed in figure 17. The optimal inflow disturbance is first reached in five ascent iterations (indicated in the figure by circles), and its optimal control is evaluated in the successive four descent iterations (indicated by crosses). Afterwards the inset shows that the saddle-point solution is reached by alternating an ascent and a descent iteration. Convergence to the saddle point is achieved in this case very rapidly, whereas for other strategies a greater number of iterations may be necessary. However, the achieved convergence provides a strong argument in favour of the algorithm first put forward by Bewley and co-workers.

The 'robust' control strategy has been applied to steady inflow disturbances and the results are shown in figures 18, 19 and 20. The magnitude of the disturbance energy $E_{u}$ resulting from robust-control calculations is higher than the corresponding optimal-control energies. This should be the case since the optimal disturbance can now 'feel' the presence of the wall forcing and adjusts itself so as to further maximize the cost functional $\mathscr{I}$. The difference increases with the magnitude of the control, quantified by $E_{c_{0}}$. Indeed, as can be seen from figure 18, a discrepancy between 'robust' and 'optimal' control is hardly visible in the presence of the 'cheap' control strategy, the control energy of which is the lowest. The difference is more pronounced for the other strategies, especially for the 'small' one that has the highest value for the control energy. Figure 19 shows that the profiles of the control vary slightly between the optimal and robust cases with the difference being more pronounced for the 'flat' 


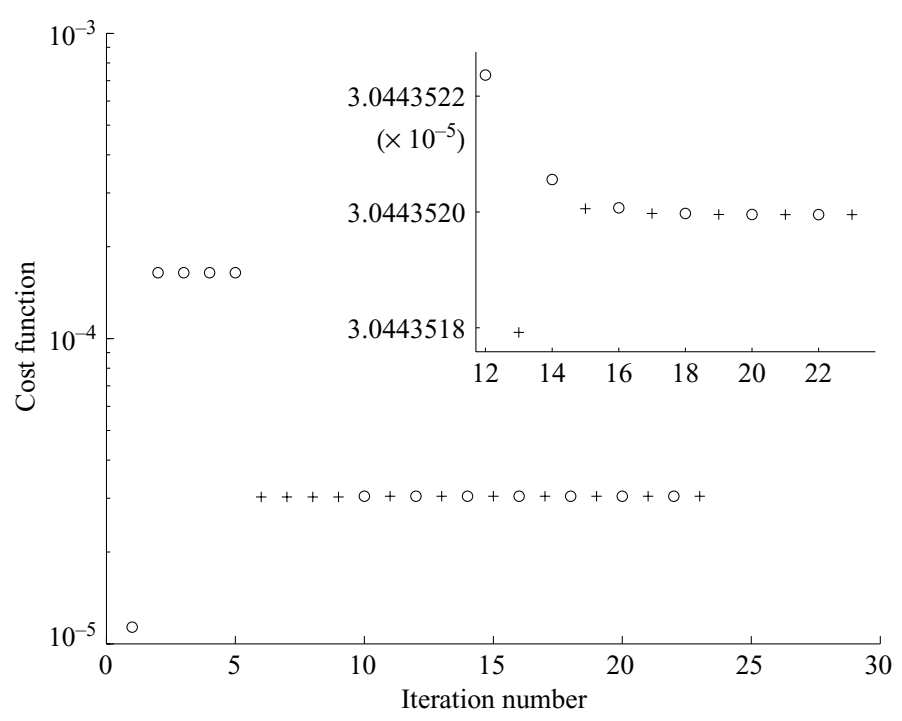

FIGURE 17. Value of the cost $\mathscr{I}$ as a function of the iteration number for the 'cheap' strategy. $\bigcirc$, ascent iterations; + , descent iterations.
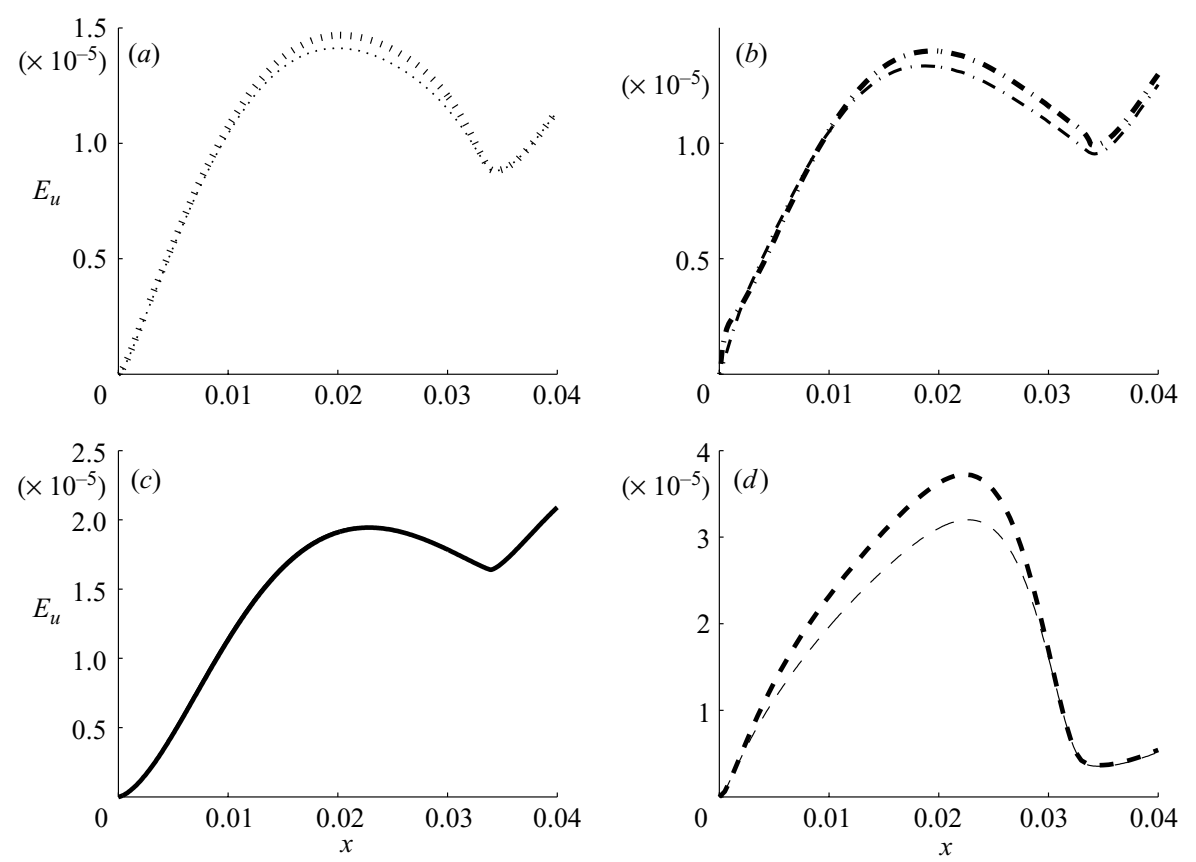

FIGURE 18. Optimal and robust growth curves for $m=1, \omega=0$, in the presence of different control strategies. (a) 'equil', $E_{c_{0}}=0.00192 ;(b)$ 'flat', $E_{c_{0}}=0.00188 ;(c)$ 'cheap', $E_{c_{0}}=0.00108$; $(d)$ 'small', $E_{c_{0}}=0.00386$. Thick lines correspond to robust control, thin lines to optimal control solutions.

strategy, in which stronger peaks are observed at the edges of the control interval. Whereas it would have been interesting to examine configurations, for which optimal and robust strategies yielded widely differing results, we are confined to a linear setting and thus cannot treat flow cases with large inflow disturbance and/or control 

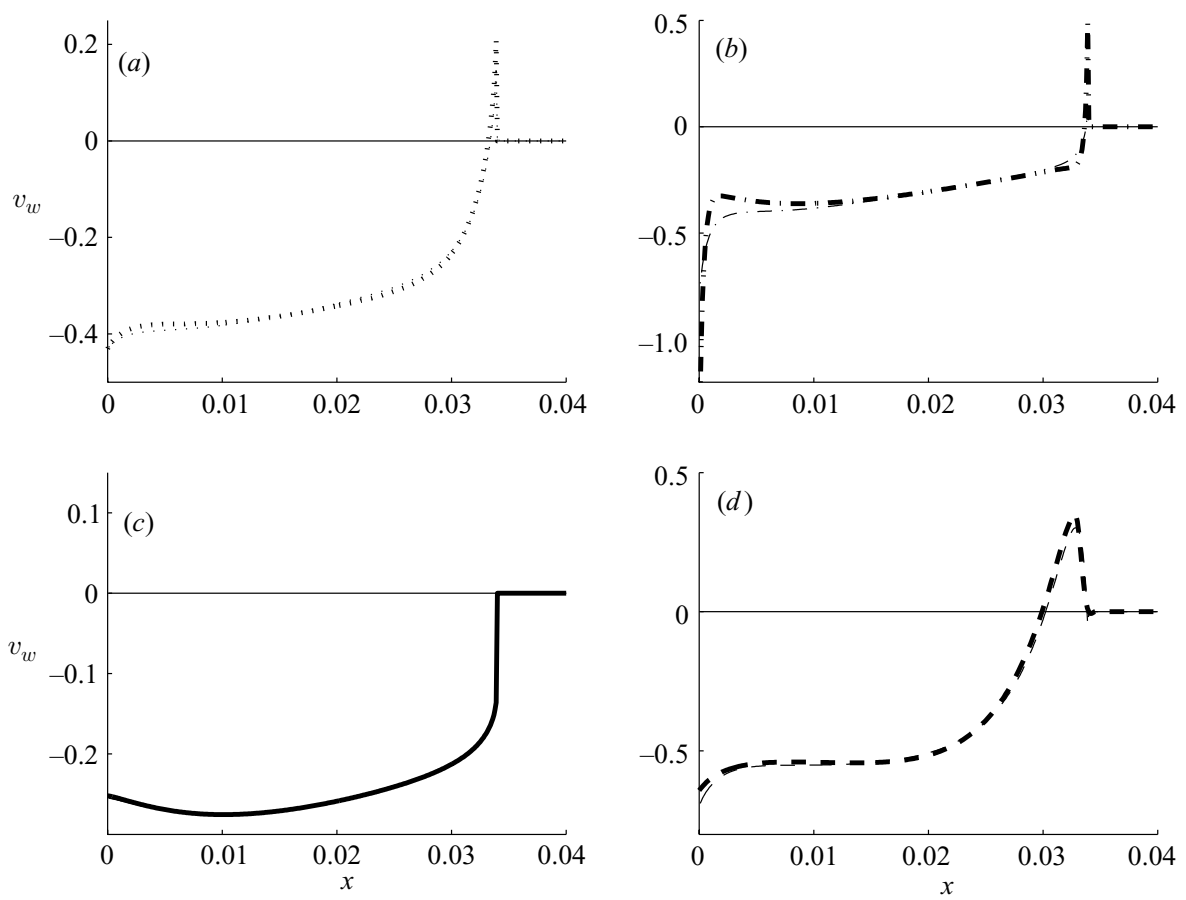

FiguRE 19. Wall velocity distributions for $m=1, \omega=0$, in presence of different control strategies. $(a)$ 'equil', $E_{c_{0}}=0.00192 ;(b)$ 'flat', $E_{c_{0}}=0.00188 ;(c)$ 'cheap', $E_{c_{0}}=0.00108$; (d) 'small', $E_{c_{0}}=0.00386$. Thick lines correspond to robust control, thin lines to optimal control solutions.

energies. Zuccher et al. (2004) have applied the same ascent-descent technique for the laminar flow control of a boundary layer with large-amplitude streaks, but even for some of the more 'extreme' configurations selected they were unable to obtain large differences between the optimal and the robust control laws computed. This is an asset for practical applications, since it suggests that optimal control strategies have some degree of validity even beyond the flow cases, for which they were designed to function.

In figure 20, we present the inflow disturbances that, for the various control strategies as given in table 1, maximize (5.1) in presence of the optimal control in the form of blowing/suction at the wall. In the robust case, the centre of the vortices is displaced towards the inner region of the pipe, where the disturbances are slightly stronger, in comparison with the case of optimal control. Again the difference is more evident for those strategies, in which the control effort is larger.

\section{Summary and conclusions}

A spatial analysis has been performed of the evolution and control of steady and mildly unsteady algebraically growing instabilities in Hagen-Poiseuille flow. For the analysis we have adopted a parabolic approximation to the full elliptic Navier-Stokes equations based on the assumption of slow streamwise variation of the flow field. This approximation is shown to perform well at the Reynolds numbers of interest for transition. 
(a)

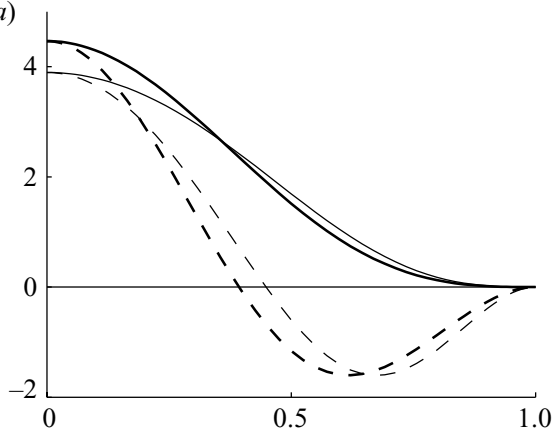

(c)

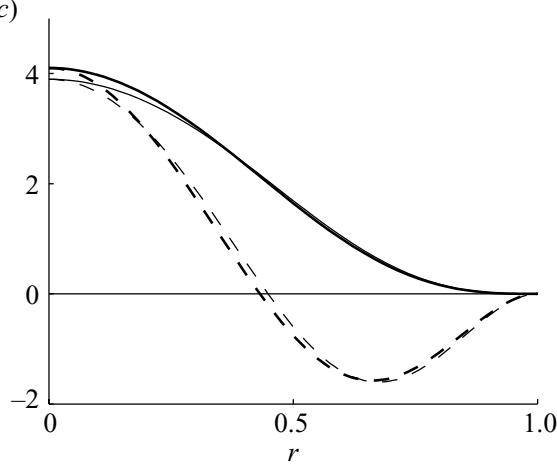

(b)

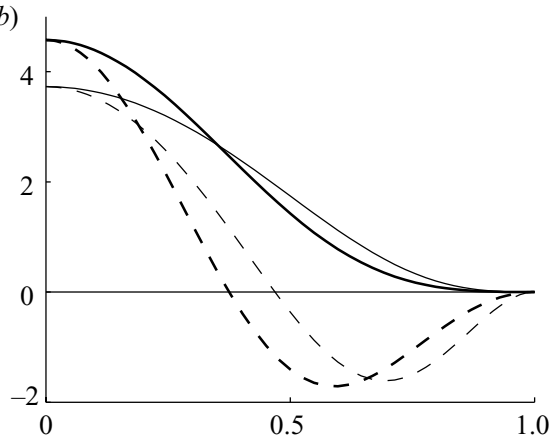

(d)

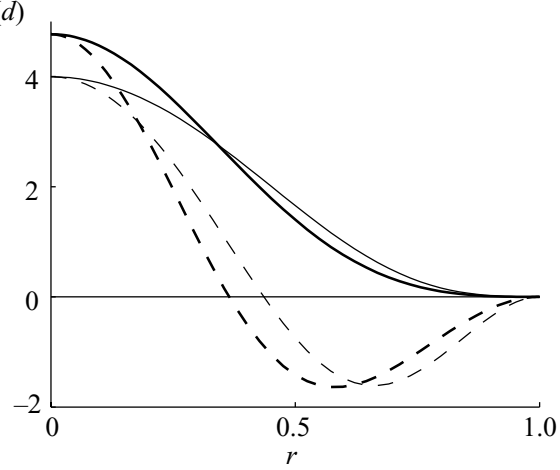

FIgURE 20. Radial (solid lines) and azimuthal (dashed lines) velocity components for the optimal and robust inflow perturbations with $m=1, \omega=0$. (a) 'equil', $E_{c_{0}}=0.00192$; $(b)$ 'flat', $E_{c_{0}}=0.00188 ;(c)$ 'cheap', $E_{c_{0}}=0.00108 ;(d)$ 'small', $E_{c_{0}}=0.00386$. Thick lines correspond to the robust case and thin lines to the optimal case.

An optimization technique based on the use of an adjoint system of equations has been applied. First, the inflow conditions that excite the largest response from Hagen-Poiseuille flow have been determined in the absence of control. These so-called optimal perturbations have the form of streamwise vortices. Subsequently, the optimal control in the form of blowing/suction at the wall, has been computed. Optimal in this context means a control that leads to the smallest growth of the perturbation kinetic energy for a given inflow condition. Finally, we have searched for the worst possible inflow perturbation in the presence of the corresponding optimal control, a procedure known as robust control. It consists of the identification of a saddle point in parameter space of the cost functional, as outlined by Bewley et al. (2000).

The control adopted in this paper can be described as cancellation control, because it counteracts directly the optimal inflow perturbation. This approach has the drawback that it is effective only if the control is in phase with the disturbance. In other words, the inflow perturbation must be accurately measured and fed to the controller.

The cost functional to be minimized consists of a weighted sum of the control effort, the disturbance growth at a final downstream position and the average perturbation energy over the control interval. Different control strategies can be tested by giving larger weight to each of these three individual contributions. Results are computed for (optimal) inflow disturbances with azimuthal wavenumber $m=1$, since these undergo the largest transient amplification in the steady and slowly oscillating cases. When optimal control is applied, an overall reduction in disturbance growth is obtained 
for all the control strategies analysed. However, we must emphasize the importance of controlling the average perturbation energy over the control interval rather than only the disturbance energy at the final position. In fact, there is sufficient evidence showing that this latter strategy can induce larger excursions in perturbation kinetic energy than those experienced in the uncontrolled case.

Steep gradients can occur in the initial distribution of the wall forcing for certain control strategies considered. However, full numerical simulations have shown that their occurrence does not contradict the underlying hypothesis of slow streamwise variation of the disturbance flow field and that the parabolic model is sufficiently accurate in describing the evolution of the disturbance under active control conditions.

Physically, the effectiveness of the control in the form of wall blowing/suction distributions in attenuating the disturbance growth, relies upon the displacement of the streamwise vortices, which constitute the optimal perturbations, away from the wall. As a consequence, the lift-up mechanism, that is at the basis of transient growth of elongated streaks, becomes less effective. This interpretation is entirely consistent with the mothers-daughters scenario suggested by Boberg \& Brosa (1988) and which we discussed in $\S 1$. Our results confirm their deduction that 'ruling mothers means ruling turbulence'. Indeed, the growth of the streaks (the daughters), which dominate the appearance of turbulent and transitional flows, can be neutralized by acting on the streamwise vortices (the mothers).

In the uncontrolled case, the presence of strong streaks provides the flow with the potential to become unstable, because saddle points appear in the axial velocity profile that could undergo an inviscid secondary instability with respect to three-dimensionaldisturbances (see for instance Zikanov 1996; Meseguer 2003). Controlling the size of the streaks could therefore result in a spatial delay of the transition process. However, the full numerical simulations show that the linear control laws are effective only if the flow structures do not deviate too much from the streamwise elongated streaks arising from a linear evolution and for which the optimal control has been computed. This is a limitation of the cancellation control approach adopted in this paper. When the nonlinear interactions become important, the flow field is essentially three dimensional and the optimal control laws must result from full nonlinear calculations (see Zuccher et al. 2004).

In practical applications, control will most likely be feasible only at a limited number of sections along the pipe. Therefore, we have also investigated the effect of control along a short window or along two consecutive windows. Such a local control will always be less efficient than the case in which the wall forcing is applied from the inflow section up to the streamwise location of maximum growth in the uncontrolled case. Nonetheless, a consistent reduction in perturbation kinetic energy is observed that is optimal when the axial location of the control window is chosen such that the control attains equal values at its edges.

In addition, robust control calculations have been carried out to investigate the influence of the applied wall forcing on the optimal inflow perturbation. Since this leads to the worst possible inflow condition in combination with the control, the resulting disturbance energy curves are always above those of the optimal control case with a fixed inflow condition. As expected, the difference increases with the control energy since this results in a stronger modification of the flow field. Nevertheless, we found that this difference always remains small since the relatively weak control efforts considered are not sufficient to alter the flow field very strongly.

We believe that we have been the first to address the problem of the optimal control of laminar streaks in pipe flow. Although it has provided us with much insight into 
effective strategies to damp streaks, the technique employed has the limitation of being open-loop, i.e. new calculations are required for all different inflow conditions imposed. On the other hand, the fact of having developed a parabolic framework for the definition of the state of the system, renders this flow amenable to feedback control with the ingenious procedure recently laid out and tested by Cathalifaud \& Bewley (2004a); Cathalifaud \& Bewley (2004b).

The authors wish to thank an anonymous reviewer for useful remarks on the scaling of pressure, as discussed in $\S 2$. The first author wishes to express her gratitude to Professor Nieuwstadt for his precious guidance and inspiration during her Ph.D. studies.

\section{REFERENCES}

Abergel, F. \& Temam, R. 1990 On some control problems in fluid mechanics. Theoret. Comput. Fluid Dyn. 1, 303-325.

Airiau, C., Bottaro, A., Walther, S. \& Legendre, D. 2003 a methodology for optimal laminar flow control: Application to the damping of Tollmien-Schlichting waves in a boundary layer. Phys. Fluids 15, 1131-1145.

Andersson, P., Berggren, M. \& Henningson, D. S. 1999 Optimal disturbances and bypass transition in boundary layers. Phys. Fluids 11, 134-150.

Bergström, L. 1993 Optimal growth of small disturbances in pipe Poiseuille flow. Phys. Fluids A 5, 2710-2720.

Bewley, T. R. \& LiU, S. 1998 Optimal and robust control and estimation of linear paths to transition. J. Fluid Mech. 365, 305-349.

Bewley, T. R., Temam, R. \& Ziane, M. 2000 A general framework for robust control in fluid mechanics. Physica D 138, 360-392.

Boberg, L. \& Brosa, U. 1988 Onset of turbulence in a pipe. Z. Naturforsch. 43a, 697-726.

Butler, K. M. \& FARrell, B. 1992 Three-dimensional optimal perturbations in viscous shear flow. Phys. Fluids A 4, 1637-1650.

Cathalifaud, P. \& Bewley, T. 2004a A noncausal framework for model-based feedback control of spatially developing perturbations in boundary-layer flow systems. Part i: Formulation. Syst. Control Lett. 51, 1-13.

Cathalifaud, P. \& Bewley, T. $2004 b$ A noncausal framework for model-based feedback control of spatially developing perturbations in boundary-layer flow systems. Part ii: Numerical simulations using state feedback. Syst. Control Lett. 51, 15-22.

Cathalifaud, P. \& Luchini, P. 2000 Algebraic growth in boundary layers: optimal control by blowing and suction at the wall. Eur. J. Mech. Fluids B 19, 469-490.

Corbett, P. \& Bottaro, A. 2001a Optimal control of nonmodal disturbances in boundary layers. Theoret. Comput. Fluid Dyn. 15, 65-81.

Corbett, P. \& Bottaro, A. $2001 b$ Optimal linear growth in swept boundary layers. J. Fluid Mech. 435, $1-23$.

Eggels, J. G. M., Unger, F., Weiss, M. H., Westerweel, J., Adrian, R. J., Friedrich, R. \& Nieuwstadt, F. T. M. 1994 Fully developed turbulent pipe flow: a comparison between direct numerical simulation and experiment. J. Fluid Mech. 268, 175-209.

FARrell, B. 1988 Optimal excitation of perturbations in viscous shear flow. Phys. Fluids 31, 2093-2102.

Gavarini, M. I., Bottaro, A. \& Nieuwstadt, F. T. M. 2004 The initial stage of transition in pipe flow: role of optimal base-flow distortions. J. Fluid Mech. 517, 131-165.

Gill, A. E. 1965 A mechanism for instability of plane Couette flow and of Poiseuille flow in a pipe. J. Fluid Mech. 21, 503-511.

GunZBURGER, M. D. 1997 Introduction into mathematical aspects of flow control and optimization. In Inverse design and optimization methods, pp. 1-20, von Kármán Institute for Fluid Dynamics Lecture Series Number 1997-05. Von Kármán Institute, Brussels. 
Gad-el HaK, M. 2000 Flow Control. Passive, Active, and Reactive Flow Management. Cambridge University Press.

Landahl, M. T. 1980 A note on an algebraic instability of inviscid parallel shear flow. J. Fluid Mech. 98, 243-251.

LUCHINI, P. 1996 Reynolds-number-independent instability of the boundary layer over a flat surface. J. Fluid Mech. 327, 101-115.

LUCHINI, P. 2000 Reynolds-number-independent instability of the boundary layer over a flat surface: optimal perturbations. J. Fluid Mech. 404, 289-309.

LuChini, P. \& BotTaRo, A. 1998 Görtler vortices: a backward-in-time approach to the receptivity problem. J. Fluid Mech. 363, 1-23.

Meseguer, A. 2003 Streak breakdown instability in pipe Poiseuille flow. Phys. Fluids 15, 1203-1213.

Milling, R. 1981 Tollmien-Schlichting wave cancellation. Phys. Fluids 24, 979-981.

Press, W. H., Teukolsky, S. A., Vetterling, W. T. \& Flannery, B. P. 1992 Numerical Recipes in $C, 2$ nd edn. Cambridge University Press, Cambridge.

Reshotкo, E. \& Tumin, A. 2001 Spatial theory of optimal disturbances in a circular pipe flow. Phys. Fluids 13, 991-996.

Schmid, P. J. \& Henningson, D. S. 1994 Optimal energy density growth in Hagen-Poiseuille flow. J. Fluid Mech. 277, 197-225.

Schmid, P. J. \& Henningson, D. S. 2001 Stability and Transition in Shear Flows. Springer.

Thomas, A. 1983 The control of boundary layer transition using a wave superposition principle. J. Fluid Mech. 137, 233-250.

Trefethen, L. N., Trefethen, A. E., Reddy, S. C. \& Driscoll, T. A. 1993 Hydrodynamic stability without eigenvalues. Science 261, 578-584.

Zhou, K. \& Doyle, J. 1998 Essentials of Robust Control. Prentice-Hall, Englewood Cliffs, NJ.

Zikanov, O. Y. 1996 On the instability of pipe Poiseuille flow. Phys. Fluids 8, 2923-2932.

Zuccher, S., Luchini, P. \& Bottaro, A. 2004 Algebraic growth in a Blasius boundary layer: optimal and robust control by mean suction in the nonlinear regime. J. Fluid Mech. 513, $135-160$. 